\title{
On the Robustness of Conceptual Rainfall-Runoff Models to Calibration and Evaluation Dataset Splits Selection: A Large Sample Investigation
}

\section{Danlu Guo ${ }^{1,2}$, Feifei Zheng ${ }^{2}$, Hoshin Gupta ${ }^{3}$ and Holger R. Maier ${ }^{2,4}$}

${ }^{1}$ Department of Infrastructure Engineering, The University of Melbourne, Parkville, VIC Australia

${ }^{2}$ College of Civil Engineering and Architecture, Zhejiang University, Hangzhou, Zhejiang, China

${ }^{3}$ Department of Hydrology and Atmospheric Sciences, The University of Arizona, Tucson, AZ, USA

${ }^{4}$ School of Civil, Environmental and Mining Engineering, University of Adelaide, Adelaide, SA Australia

Corresponding author: Feifei Zheng (feifeizheng@zju.edu.cn)

\section{Key Points:}

- We investigate the robustness of CRR models across calibration/evaluation data splits with a large-sample approach.

- Different data splits markedly impact CRR model performance, particularly for accurately reproducing the mean and variability of runoff.

- Low performance robustness is related to high runoff skewness and aridity, variable baseflow contribution, and low rainfall-runoff ratio.

This is the author manuscript accepted for publication and has undergone full peer review but has not been through the copyediting, typesetting, pagination and proofreading process, which may lead to differences between this version and the Version of Record. Please cite this article as doi: 10.1029/2019WR026752 


\begin{abstract}
Conceptual Rainfall-Runoff (CRR) models are widely used for runoff simulation, and for prediction under a changing climate. The models are often calibrated with only a portion of all available data at a location, and then evaluated independently with another part of the data for reliability assessment. Previous studies report a persistent decrease in CRR model performance when applying the calibrated model to the evaluation data. However, there remains a lack of comprehensive understanding about the nature of this 'low transferability' problem and why it occurs. In this study we employ a large sample approach to investigate the robustness of CRR models across calibration/validation data splits. Specially, we investigate: 1) how robust is CRR model performance across calibration/evaluation data splits, at catchments with a wide range of hydro-climatic conditions; and 2) is the robustness of model performance somehow related to the hydro-climatic characteristics of a catchment? We apply three widely used CRR models, GR4J, AWBM and IHACRE_CMD, to 163 Australian catchments having long-term historical data. Each model was calibrated and evaluated at each catchment, using a large number of data splits, resulting in a total of 929,160 calibrated models. Results show that: 1) model performance generally exhibits poor robustness across calibration/evaluation data splits; 2) lower model robustness is correlated with specific catchment characteristics, such as higher runoff skewness and aridity, highly variable baseflow contribution, and lower rainfall-runoff ratio. These results provide a valuable benchmark for future model robustness assessments, and useful guidance for model calibration and evaluation.
\end{abstract}

\title{
Plain Language Summary
}

Conceptual Rainfall-Runoff (CRR) models are widely used to model historical streamflow and to make predictions. These model structures are often determined through calibration to a portion of available data, with an independent data portion used for reliability evaluation. While several studies have noted decreasing performance when applying the calibrated model to the evaluation data, a comprehensive understanding of this issue and its causes is lacking.

This study uses a large number of catchments to investigate the robustness of CRR models to different ways of data split for calibration/evaluation, specifically: 1) how robust is model performance across data splits, for catchments subject to various rainfall, runoff and other climatic conditions; and 2) is this robustness related to the catchment properties? We applied three widely-used CRR models to 163 Australian catchments having long-term historical data, considering numerous calibration/evaluation data splits which led to 929,160 calibrated models. We found: 1) model performance generally lacks robustness across data splits; 2) model robustness is lower at catchments with higher runoff skewness and aridity, higher variation in baseflow contribution, and lower rainfall-runoff ratio. This study provides a valuable benchmark for future model robustness assessments, and can also inform strategies for model calibration and evaluation. 


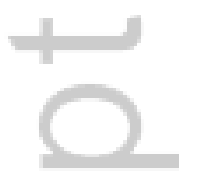

4 


\section{Introduction}

[1] Rainfall-runoff models are widely used to simulate catchment runoff, estimate risks of droughts and floods, and more recently to estimate the local and regional impacts of global climate change [e.g. Boughton \& Droop, 2003; Gibbs et al., 2012; Jones et al., 2006; Kay et al., 2006; Najafi \& Moradkhani, 2015; Vaze \& Teng, 2011; Zheng et al., 2015]. These models can be generally categorized into empirical, conceptual and physical models [Beven, 2011]. However, due to limitations in existing knowledge and information on the complex mechanisms that drive hydrological systems, even the most physically-based rainfall-runoff model remains conceptual or empirical to some extent [Mount et al., 2016; Murphy et al., 2006], and parameter estimation through model calibration is therefore typically necessary. Further, to understand the capacity of the model to predict runoff for periods other than the calibration period, it is standard practice to assess the performance of the calibrated model using an independent evaluation period [Beven, 1989; KlemeŠ, 1986]. Therefore, the parameterization of a rainfall-runoff model is essentially determined by calibration data, while model performance is assessed with the evaluation data.

[2] In general, when developing a rainfall-runoff model, the total available data are divided into two subsets for model calibration and evaluation, and hence the results of the performance evaluation can depend on the specific data split selected for carrying out these two steps [Wu et al., 2013; Zheng et al., 2018]. This dependence on data split seems to especially be the case if the hydro-climatic conditions are highly variable over time. For instance, if the calibration period contains a number of extreme rainfall events, while such events are rarely observed in the evaluation period, the calibrated model may perform well under hydro-climatic conditions characterized by frequent extreme events, but perform poorly on a period having much lower frequency of such extreme events. This phenomenon has been referred to as the 'low transferability' of rainfall-runoff models, where model performance declines when the calibrated model is used to simulate the behaviour of periods other than the calibration period [Hartmann \& Bárdossy, 2005].

[3] The issue of low transferability across calibration and evaluation periods when dealing with contrasting hydro-climatic conditions has been increasingly discussed over the past decade, mainly in the context of conceptual rainfall-runoff (CRR) models [e.g. Bastola et al., 2011; Broderick et al., 2016; Coron et al., 2012; Gibbs et al., 2018; Li et al., 2012]. A few studies have investigated how model performance can be influenced by contrasting conditions between the calibration and evaluation datasets. For example, Vaze et al. [2010] compared the performance of four commonly used CRR models (SIMHYD, Sacramento, SMARG and IHACRE) and reported consistently worse model performance when simulations conditions were drier than the calibration period. Bastola et al. [2011] and Coron et al. [2012] also focused on CRR models and found that the decline in evaluation period performance relative to the calibration period could be related to differences in average rainfall intensity across the two periods. Further, a few approaches attempting to resolve this CRR model low-transferability problem have been proposed. For example, Bastola et al. 
[2011] suggested the use of a sufficiently long calibration period that is representative of a wider range of dry and wet conditions, consistent with earlier recommendations by Sorooshian et al. [1983], Gupta and Sorooshian [1985] and Yapo et al. [1996]. In addition, Li et al. [2012] and Gibbs et al. [2018] suggested that calibration should be performed for a sub-period of the full data which has similar hydro-climatic conditions to those expected for the evaluation period.

[4] While the impact of data split selection on CRR model performance has received recent attention in the literature, relevant studies have been largely ad-hoc. Specifically, the key focus has been on the relative difference in CRR model performance associated with hydroclimatic differences between the calibration and evaluation periods, with the main goal of understanding how much the performance might be expected to deteriorate when the model is used under a changing climate. However, to our knowledge, there has not been a systematic study to understand how and why the performance of CRR models varies with the selection of different data splits, when the hydro-climatic conditions under which these models are being applied vary substantially. The closest such assessment, Zheng et al. [2018], conducted a comprehensive investigation of the impact of data splits on rainfall-runoff modelling with artificial neural network (ANN) models, which consist of a data-driven (rather than conceptual) modelling approach. Using data from 754 catchments across Australia and the US, the results showed clearly that the choice of data split method (4 different methods were tested) had a significant impact on model performance, particularly for catchments where the distribution of runoff data is more highly skewed. However, due to fundamental structural differences between the ANN and CRR model types, the findings of Zheng et al. [2018] do not directly transfer to CRR models. Specifically, in the ANN models, any memory in hydroclimate conditions affecting runoff at a particular point in time (i.e. for each of the runoff values in the calibration and evaluation data sets) is represented via time-lagged values in independent input-output vectors [Maier et al., 2010], where appropriate values of these lags can be obtained via various input variable selection methods [Galelli et al., 2014]. As such, the data-driven ANN models used by Zheng et al. [2018] do not require the use of continuous time periods of data for calibration and validation. In contrast, CRR models consist of state variables that are updated according to historical hydro-climatic conditions, which makes it a key requirement to maintain the temporal structure of input and output data [e.g. Perrin et al., 2003; Wagener et al., 2003].

[5] Accordingly, this paper aims to systematically investigate the impacts of data split selection on the evaluation performance of three widely-used CRR models. Specifically, we investigate two research questions:

1) How much variability in CRR model performance can be expected across different data splits, across a large number of catchments having a wide range of hydroclimatic conditions?

2) Can the robustness of CRR model performance be tied to the hydro-climatic characteristics of catchments? 
[6] The study was conducted using three CRR models - GR4J [Perrin et al., 2003], AWBM [Boughton, 2004; Boughton, 1993; Boughton \& Carroll, 1993] and IHACRE_CMD [Croke \& Jakeman, 2004] - applied to 163 Australian catchments that span a wide range of hydro-climatic regions, and for which long-term data are available. We use a large-sample hydrology approach [Gupta et al., 2014], with the goal of achieving generalizable findings and observations [e.g. Coron et al., 2012; Gudmundsson et al., 2012; Merz et al., 2011] and model benchmarking [Newman et al., 2017]. To ensure analytical rigor, each CRR model is calibrated and evaluated using a large number of data splits at each catchment, leading to the development and testing of 929,160 model-dataset combinations. Our goal is to improve general understanding of the evaluation performance of CRR models as a function of varying data splits, and how this performance may be related to the hydro-climatic characteristics of catchments. Being the first study of this kind, these results provide a valuable benchmark for assessing the robustness of CRR models, while highlighting the importance and necessity of future improvements in calibration/evaluation methodology for CRR models.

\section{Method}

[7] We briefly describe the three CRR models in Section 2.1, and our study catchments and key datasets in Section 2.2. The models were calibrated and evaluated multiple times using a large number of different calibration/evaluation data splits (Section 2.3), and these results were analysed to investigate large-scale patterns of CRR model robustness (Section 2.4).

\subsection{The conceptual rainfall-runoff models}

[8] For this study, we focused on three widely-used daily time-step CRR models, all of which represent the catchments as being spatially-lumped, and which estimate catchmentoutlet runoff from catchment-averaged rainfall (P) and potential evapotranspiration (PET) data. The models differ in structural representation, which affects their input-state-output behaviour, which can thus lead to different levels of performance robustness. Specifically:

M1 The four-parameter GR4J model [Perrin et al., 2003] consists of a single soil moisture store, and uses continuous relationships between the moisture level in the soil store and runoff production. On a dry day where P is less than PET, the soil store level determines the proportion of PET that contributes to actual evapotranspiration (AET). On a wet day (P exceeding PET), the soil store level determines the proportion of net rainfall ( $\mathrm{P}$ minus $\mathrm{PET}$ ) that replenishes the soil moisture store, while the remaining becomes effective rainfall that contributes to runoff production. A unit hydrograph is used for runoff routing. The four free parameters of the model are: soil moisture store capacity, groundwater exchange rate, one-day runoff production store capacity, and time-base for unit hydrograph.

M2 The four-parameter AWBM model [Boughton, 2004; Boughton, 1993; Boughton \& Carroll, 1993] conceptualizes the catchment as having three soil moisture stores of increasing capacity. As each store is filled with net rainfall, it starts contributing to 
runoff production when the store moisture content exceeds its capacity. This study used the self-calibrated version of the model, AWBM2002, which applies constant fraction areas of each store (see Boughton [2004] for details) and thus uses a single parameter to define the properties of the soil moisture store (i.e., area-averaged store capacity). Therefore, along with three other parameters used for linear runoff-routing, the AWBM model applied in this study has a total of four free parameters.

M3 The five-parameter IHACRES-CMD model [Croke \& Jakeman, 2004] (hereafter called CMD) partitions net rainfall in a manner similar to GR4J, but differs by using the moisture deficit in the soil moisture store to determine such partitioning. The model uses two parameters to define the threshold soil moisture store levels to produce runoff and to turn off AET. The linear routing model is essentially the same as that for AWBM, which has three parameters. Therefore, this model has a total of five free parameters.

[9] All three models have previously been implemented in an R package called hydromad (http://hydromad.catchment.org/) [Andrews \& Guillaume, 2013; Andrews et al., 2011]. For all of the calibration runs (Section 2.3), we used the Shuffled Complex Evolution (SCE) global optimization procedure, with the Kling-Gupta Efficiency (KGE) as the performance metric [Gupta et al., 2009; Kling et al., 2012]. KGE, as shown below, summarizes three key aspects of performance for a rainfall-runoff model, specifically: 1) simulating the mean; 2) simulating the variability 3) maintaining the serial correlation, of the observed runoff timeseries:

$$
\begin{array}{r}
K G E=1-\sqrt{(r-1)^{2}+(\beta-1)^{2}+(\gamma-1)^{2}} \\
\beta=\frac{\mu_{\text {sim }}}{\mu_{o b s}} \\
\gamma=\frac{C V_{\text {sim }}}{C V_{o b s}}=\frac{\sigma_{\text {sim }} / \mu_{\text {sim }}}{\sigma_{o b s} / \mu_{\text {sim }}}
\end{array}
$$

where $r$ is the correlation coefficient between simulated and observed runoff, $\beta$ is the ratio of simulated to observed mean runoff (i.e. bias), and $\gamma$ is the ratio of simulated to observed runoff variability, as represented by the coefficient of variation $(\mathrm{CV})$ of each time series, respectively [Gupta et al., 2009; Kling et al., 2012]. The optimal value for each of $r, \beta$ and $\gamma$ is 1 , which is achieved when the simulated and observed time series are identical. Accordingly, KGE can range from negative infinity to 1 , and SCE is implemented so as to maximize its value with respect to the model parameters.

\subsection{Data and pre-screening analyses}

[10] To investigate the performance robustness of the three aforementioned models, we considered the full set of 221 available hydrologic reference stations (HRS) across Australia. These stations consist of high-quality historical daily runoff records at individual catchment outlets, provided by the Australian Bureau of Meteorology (via 
http://www.bom.gov.au/water/hrs/). After extracting all available runoff records for these catchments, we narrowed the selection to 209 catchments, for which more than 30 years of historical data are available. Our intention was to ensure that our study sampled a wide range of hydro-climatic conditions when calibrating and evaluating the models at each catchment (see Section 2.3). To drive the models, we used catchment-average rainfall and PET, extracted from the Australian Water Availability Project (AWAP) gridded dataset [Raupach et al., 2009, 2012] together with catchment boundaries extracted by Fowler et al. [2016].

[11] We then pre-screened the catchments to identify catchments where the models were likely to provide relatively stable performance with minimal uncertainty due to the calibration procedure. Specifically, the model performance should be less variable across individual calibration runs when starting from different random seeds, which represent different random start positions within the parameter space from where the optimization process begins searching [Maier et al., 2019]. To do this, we calibrated GR4J to the entire available period of record for each of the 209 catchments, and repeated each calibration 100 times with different initial seeds for the SCE algorithm, thereby assessing model uncertainty due to implementation of the optimization method. Note that a previous review of CRR models by Shin et al. [2015] suggested that, simple models such as GR4J are generally identifiable, with multiple calibration replicates converging to the single global optimal parameter set. However, our decision to use multiple runs for each calibration was made considering: 1) the potentially decreased ability of the SCE algorithm used for calibration in this study, as opposed to the more computationally intensive differential evolution adaptive Metropolis (DREAM) used in Shin et al. [2015]); 2) the much larger number of catchments (209) and more diverse catchment characteristics included, as opposed to the five catchments used in Shin et al. [2015].

[12] Based on this pre-screening step, we retained only the catchments where:

1) Both the average and median KGE values from the 100 replicates exceeded 0.75 ;

2) The range of the $95 \%$ confidence intervals on KGE was less than $3 \%$ of the corresponding average value.

[13] The first condition helps to ensure that a reasonable level of CRR model performance is achievable at a catchment (i.e., that the models are at least structurally consistent with the available catchment data). The second helps to ensure that uncertainty due to implementation of the calibration method is small. Within all 209 catchments, a subset of 163 catchments met these two conditions, for which the minimum length of data record was 33 years. A summary of the KGE performance (averages, medians and variabilities) obtained for all 209 catchments is presented in Figure S1 of the Supplementary Information. The average KGE performance for the 163 selected catchments is shown in Figure 1, superimposed on the Australian Köppen climate classifications [Stern et al., 2000], which illustrates the wide range of climatic conditions spanned by the catchments. Figure 2 summarizes the ranges and distributions of the catchment areas and key hydro-climatic conditions of the 163 catchments. 


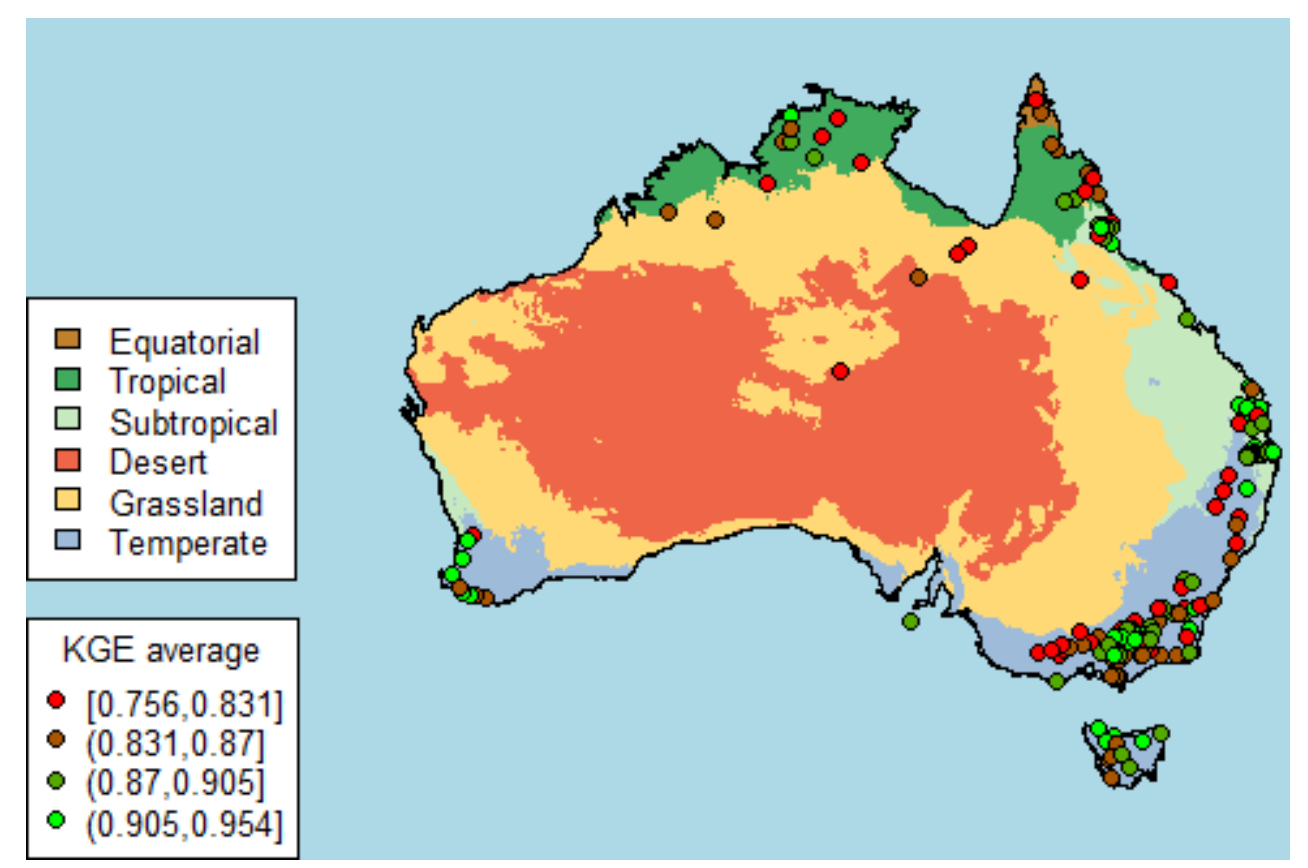

Figure 1. The 163 selected study catchments (in dots) in Australia, with colors indicating the inter-quartiles of KGE values across all catchments. At each catchment, the full record period was used for calibration, and the averaged KGE across 100 SCE replicates was used. Background map colors show the Australian Köppen climate classifications.
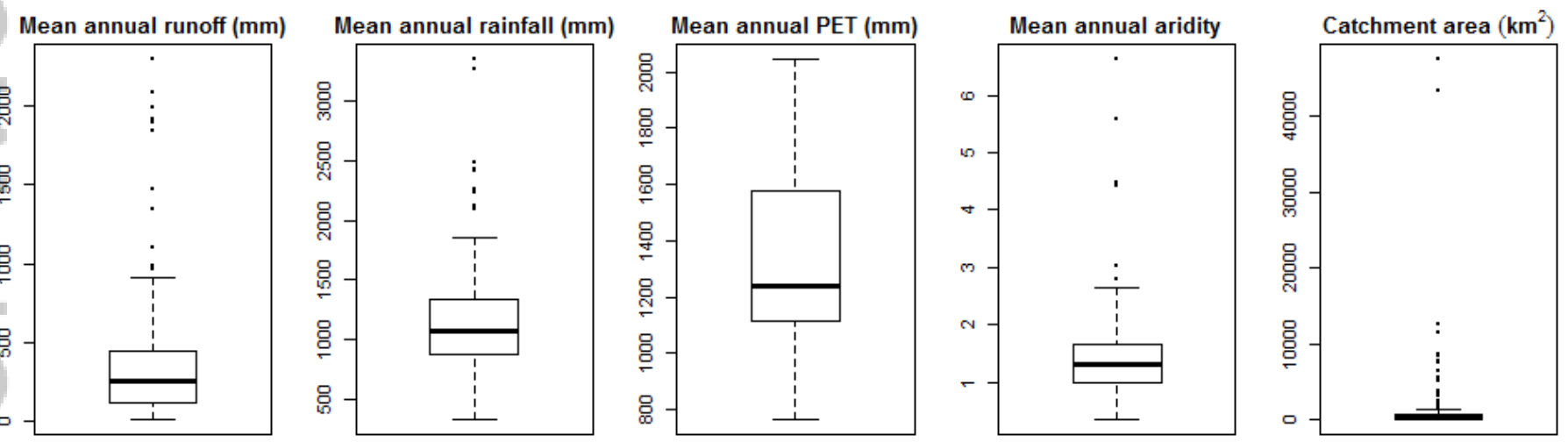

Figure 2. Summary of areas and key hydro-climatic characteristics for the 163 selected catchments. For clearer visualization, catchment areas are plotted with a logged y-axis. Aridity is defined as the PET/P ratio.

\subsection{Split-sample calibration/evaluation test}

[14] To investigate model performance robustness when calibrated under a variety of hydrometeorological conditions, we applied the generalized split-sample calibration procedure [Coron et al., 2014] to each catchment. In this approach, the full available period of record 
for each catchment was first partitioned into a large number of subperiods. Specifically, for catchment $i$ having a record length of $n_{i}$ years, we identified all time-continuous subperiods of $n_{i} / 2$ years' length ( $50 \%$ of the available data), where the starting dates of any two consecutive subperiods are separated by three months. Each block of these $n_{i} / 2$ years' data was used for model calibration, with the remaining $n_{i} / 2$ years' data used for evaluation, as illustrated in Figure 3. This results in a total of 15,486 data splits across the 163 catchments, each consisting of different combinations of calibration (50\%) and evaluation (50\%) data.

[15] Each model-subperiod-catchment calibration was repeated 20 times using different initial random seeds for the SCE method, to allow for calibration uncertainty (see justification for using multiple calibration replicates in Section 2.2). Accordingly, a total of 929,160 $(15,486 \times 20 \times 3)$ calibrated catchment 'models' (model-subperiod-catchment combinations) were developed and then evaluated with the corresponding independent data. A 365-day warm-up period was used in all calibration and evaluation simulations.

[16] To address the problem of 'non-continuous' evaluation periods (e.g. see second row shown in Figure 3, where the evaluation period consists of two non-continuous periods of time), each evaluation involved running the calibrated model for the entire $n_{i}$ years of available data, and then computing the evaluation period performance metrics using only the $n_{i} / 2$ years' data corresponding to the evaluation period.

Split-sample Calibration

Calibration split \#1, $n / 2$ years Evaluation split\#1, $n / 2$ years
$\underbrace{}_{\text {Calibration split \#2, } n / 2 \text { years Evaluation split \#2, } n / 2 \text { years }}$ months

Evaluation split \#k, $n / 2$ years Calibration split \#k, $n / 2$ years

Figure 3. Schematic of the split-sample calibration approach used for each CRR model at each catchment. Blue bars represent calibration datasets while grey bars represent evaluation sets (bar lengths are not to scale).

\subsection{Assessment of model robustness}

[17] Our assessment of CRR model robustness is based on values of KGE and its three components, $r, \beta$ and $\gamma$, as defined in Section 2.1. Specifically, we investigated: 
1) Variations of KGE and its three components across all calibration/evaluation splits and at all catchments (Section 3.1).

2) Potential relationships between model robustness and the hydro-climatic characteristics of individual catchments (Section 3.2).

[18] For the latter, specifically, 12 key catchment attributes were chosen to characterize the average conditions, variability and other important features of rainfall, runoff, evapotranspiration and topography that could potentially influence performance of conceptual rainfall-runoff models (Table 1). Of these, the Mean_runoff, Mean_rainfall, Mean_PET, Mean_BFI, Mean_aridity and Mean_RR_ratio are all estimated from annual average values, which represent the long-term conditions of runoff (including baseflow contribution), rainfall, PET and relationships among them. Meanwhile, SD_BFI, SD_aridity and $S D \_R R \_r a t i o$ focus on the short-term variability of baseflow contribution, and that of relationships between runoff, rainfall and PET, and SD_runoff and Skew_runoff summarize the day-to-day variability in streamflow and the normality of its distribution. Finally, Catchment_area was included as a key factor that influences the conversion from rainfall to runoff. It is worth noting that to obtain characteristics related to RR (rainfall-runoff) ratio and $\mathrm{BFI}, \mathrm{RR}$ ratio and BFI were first estimated at an annual time-step before computing further statistics. This is due to the high intermittency in many Australian catchments (with days having zero rainfall and runoff), which lead to substantial noise if these ratios are estimated at a daily step.

Table 1. Symbology and definitions of the 12 catchment characteristics investigated for potential relationships with model robustness.

\begin{tabular}{|l|l|}
\hline Catchment characteristic & Definition \\
\hline Mean_runoff $(\mathrm{mm})$ & $\begin{array}{l}\text { Long-term annual average runoff; estimated after the average runoff for each } \\
\text { individual year was first calculated }\end{array}$ \\
\hline Mean_rainfall $(\mathrm{mm})$ & $\begin{array}{l}\text { Long-term annual average rainfall; estimated after the average rainfall for each } \\
\text { individual year was first calculated }\end{array}$ \\
\hline Mean_PET $(\mathrm{mm})$ & $\begin{array}{l}\text { Long-term annual average PET; estimated after the average PET for each individual } \\
\text { year was first calculated }\end{array}$ \\
\hline Mean_BFI & $\begin{array}{l}\text { Long-term annual average base flow index (BFI) representing the proportion of } \\
\text { baseflow within streamflow; estimated with the Lyne-Hollick filter following } \\
\text { Ladson et al. (2013) with a recommended alpha of 0.925 }\end{array}$ \\
\hline Mean_aridity & $\begin{array}{l}\text { Long-term annual average aridity; estimated after the values for each individual } \\
\text { year was first calculated as the ratio of PET to rainfall }\end{array}$ \\
\hline Mean_RR_ratio & $\begin{array}{l}\text { Long-term annual average rainfall-runoff ratio; estimated after the values for each } \\
\text { individual year was first calculated as the ratio of runoff to rainfall }\end{array}$ \\
\hline SD_BFI & $\begin{array}{l}\text { Standard deviation of annual base flow index (BFI) representing the proportion of } \\
\text { baseflow within streamflow; estimated with the Lyne-Hollick filter following } \\
\text { Ladson et al. (2013) with a recommended alpha of 0.925 }\end{array}$ \\
\hline SD_aridity & $\begin{array}{l}\text { Standard deviation of annual aridity; estimated after the values for each individual } \\
\text { year was first calculated as the ratio of PET to rainfall }\end{array}$ \\
\hline$S D \_R R \_r a t i o$ & $\begin{array}{l}\text { Standard deviation of annual rainfall-runoff ratio; estimated after the value for each } \\
\text { individual year was first calculated as the ratio of runoff to rainfall }\end{array}$ \\
\hline
\end{tabular}




\begin{tabular}{|l|l|}
\hline SD_runoff $(\mathrm{mm})$ & Standard deviation of daily runoff \\
\hline Skew_runoff $(\mathrm{mm})$ & Skewness of daily runoff \\
\hline Area $(\mathrm{km})$ & Catchment area \\
\hline
\end{tabular}

[19] The goal of the first investigation (Section 3.1) is to assess the degree of performance robustness achievable by each model in the face of natural hydro-climatic variability. To obtain a more general understanding of model robustness, this analysis focused on a qualitative evaluation of the range of variation in the values of KGE and its three components, at each catchment within individual models. The goal of the second investigation (Section 3.2) is to assess whether model performance robustness can be tied to hydro-climatic characteristics of the catchments, and whether recommendations can be provided for selecting the most robust model according to hydro-climatic conditions at a catchment. This experiment focused on quantifying and comparing statistical relationships between model robustness and catchment characteristics; therefore, robustness of each model was evaluated as the standard deviation of KGE and its three components at each catchment, to calculate correlations with the corresponding catchment characteristics.

\section{Results}

\subsection{Are CRR models capable of maintaining robust performance across different data splits?}

[20] Overall, consistent with previous studies, we found a general lack of model performance robustness across different calibration/evaluation splits, which means that the choice of data split can strongly affect the evaluation performance of CRR models. This result is summarized in Figure 4, which compares (1) the ranges of split-sample evaluation performance at each catchment (grey lines) with (2) the corresponding calibration performance (red dots), averaged across all splits for easier visualization. The maximum range of variation in KGE (top row) for GR4J, AWBM and CMD is approximately 1.6, corresponding to catchments where negative evaluation KGEs were obtained for some of the data splits (i.e. towards the left side of the plots, negative values not shown). On the other hand, the plots also show that for a few catchments (towards the right side of the plots), the evaluation period KGE is quite similar to that during calibration, and the relative variability is quite small.

[21] Similar results are seen for the KGE components, although variability is smaller for the correlation coefficient (KGE $r$ ), consistent with the fact that the temporal patterns of streamflow are largely determined by the corresponding temporal patterns of rainfall, and therefore less dependent on model structural adequacy. 

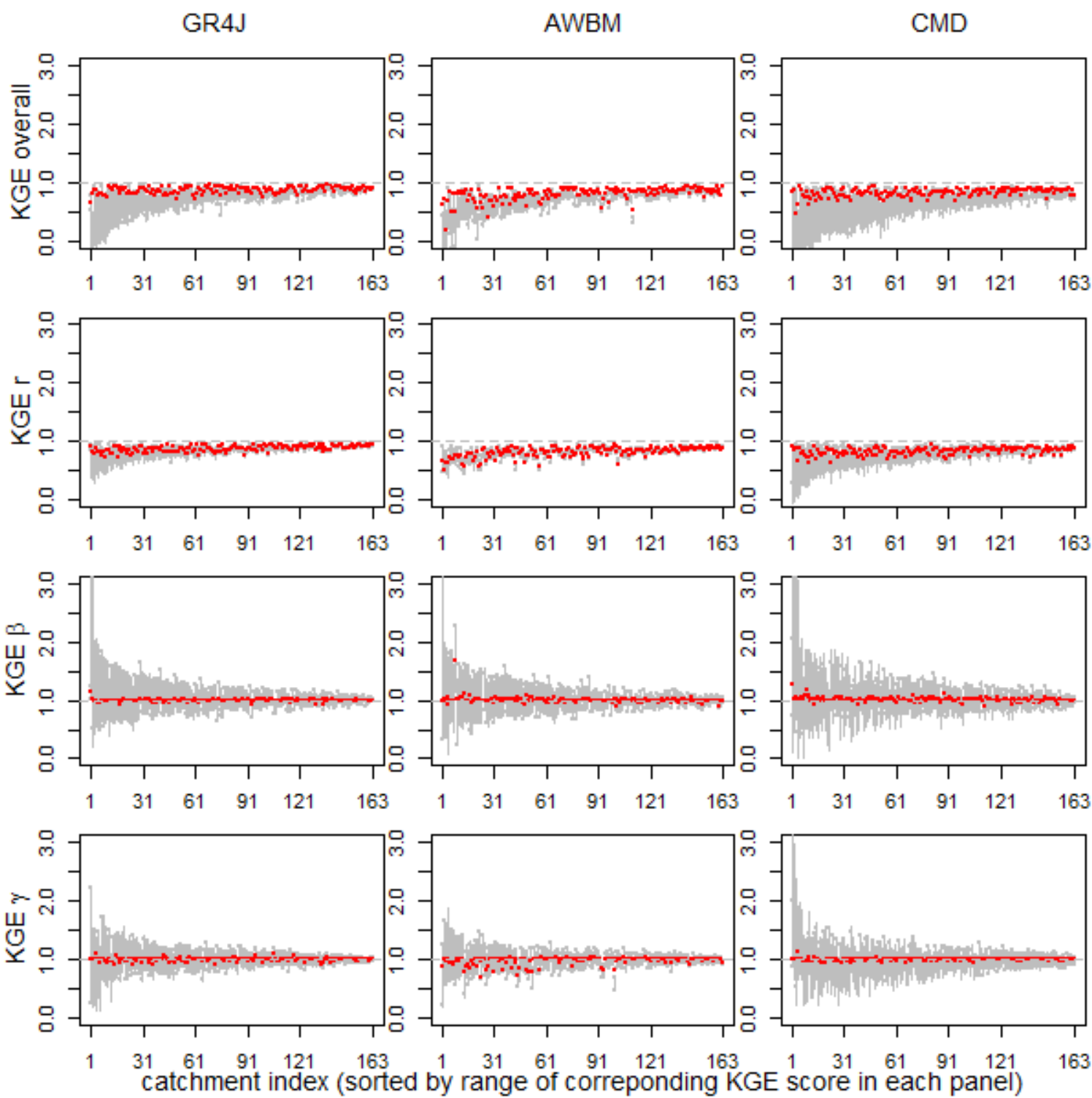

+ Average performance (calbration) — Range of performance (evaluation)

Figure 4. Model performance of GR4J, AWBM and CMD for the split-sample calibration and evaluation datasets, as summarized by the overall KGE score and its three components $(r, \beta$ and $\gamma$ ). The y-axes show the KGE metrices and the $\mathrm{x}$-axes show catchment indices. In interpreting the results, a longer grey line indicates a more highly variable evaluation performance across splits for the specific catchment i.e. lower model robustness at that catchment. For easier visualization, metric values below 0 and above 3 are not shown.

[22] Figure 5 summarizes the evaluation performance presented in Figure 4, by lumping results for each CRR model across all catchments. We see that GR4J and AWBM generally 
have more robust results (with lowest variability), while the performance robustness of the CMD model is much lower, especially as assessed in terms of KGE $r$.

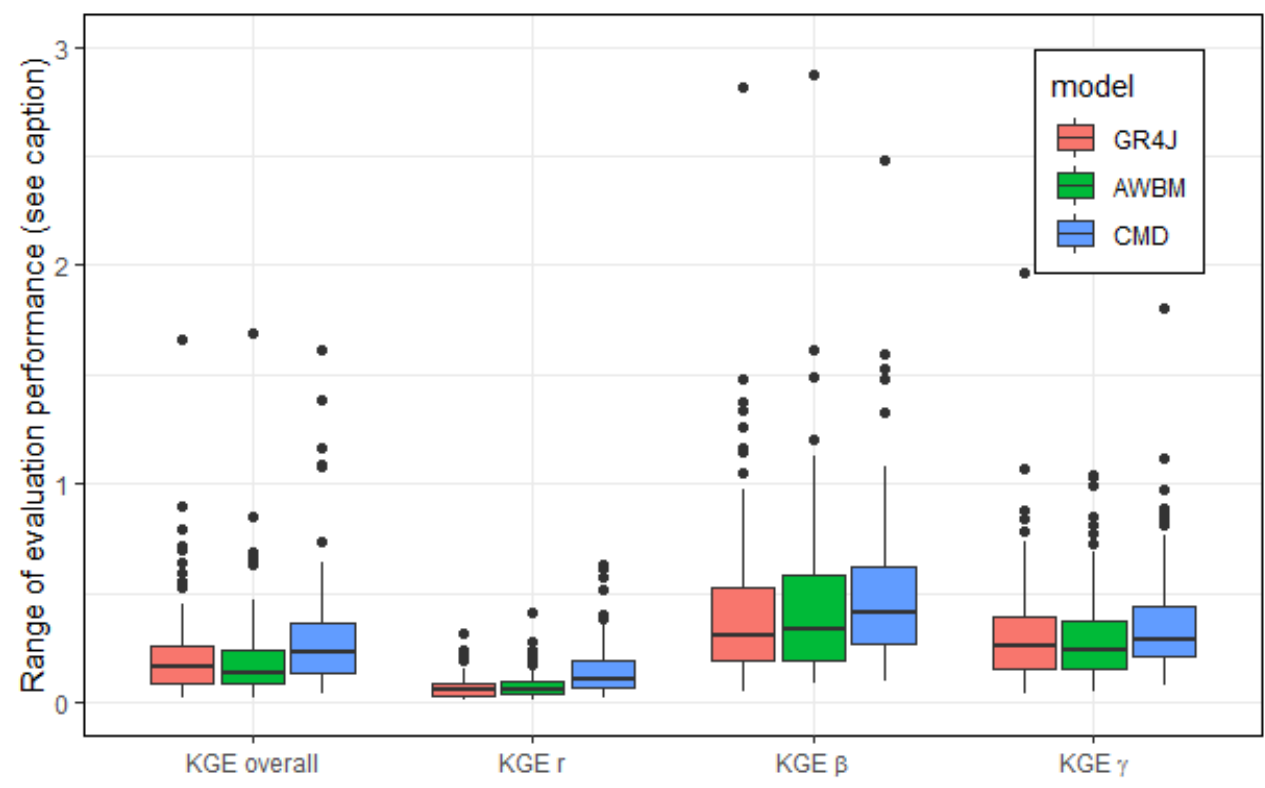

Figure 5. Ranges of evaluation performance over all calibration-evaluation data splits across all 163 catchments for GR4J, AWBM and CMD, obtained from the split-sample calibration. Performance is summarized by KGE overall, $r, \beta$ and $\gamma$, respectively, along the $\mathrm{x}$-axis. Each box shows the corresponding (grouped by model + performance metric) range of lengths of grey bars in Figure 4. For example, the 'KGE overall' box for GR4J (red) represents the range of lengths of all grey bars shown in the first panel in Figure 4. Therefore, a taller box represents a lower robustness of the model in the specific performance metric across all catchments.

[23] We also observe that performance robustness in terms of the ability to reproduce the mean and variability of runoff (KGE $\beta$ and $\gamma$ ) is lower (i.e. having higher variability in evaluation performance) compared with that for the runoff correlation (KGE $r$ ). This indicates that, when using CRR models to simulate conditions that are different from those experienced during calibration, it is more difficult to accurately predict the mean and variability of the observed runoff, compared to maintaining a high correlation with the observed time-series. As mentioned previously, this can be due to the strong correlation between runoff and the input data of rainfall.

[24] The variability of evaluation KGE $r, \beta$ and $\gamma$ is further illustrated in Figure 6 for GR4J, which shows variations in the three evaluation performance indices at each catchment against all corresponding data splits. Catchments are sorted by increasing variance of the overall KGE. As mentioned above, the correlation coefficient between simulated and observed runoff, $r$ (orange dots), generally has higher robustness, whereas the performance in 
simulating both the runoff mean and variability, $\beta$ and $\gamma$ (red and blue dots, respectively), displays high variability at a large number of catchments. Similar patterns are also seen from evaluation performances from AWBM and CMD, as in Figures A1 and A2 in the Appendix. 


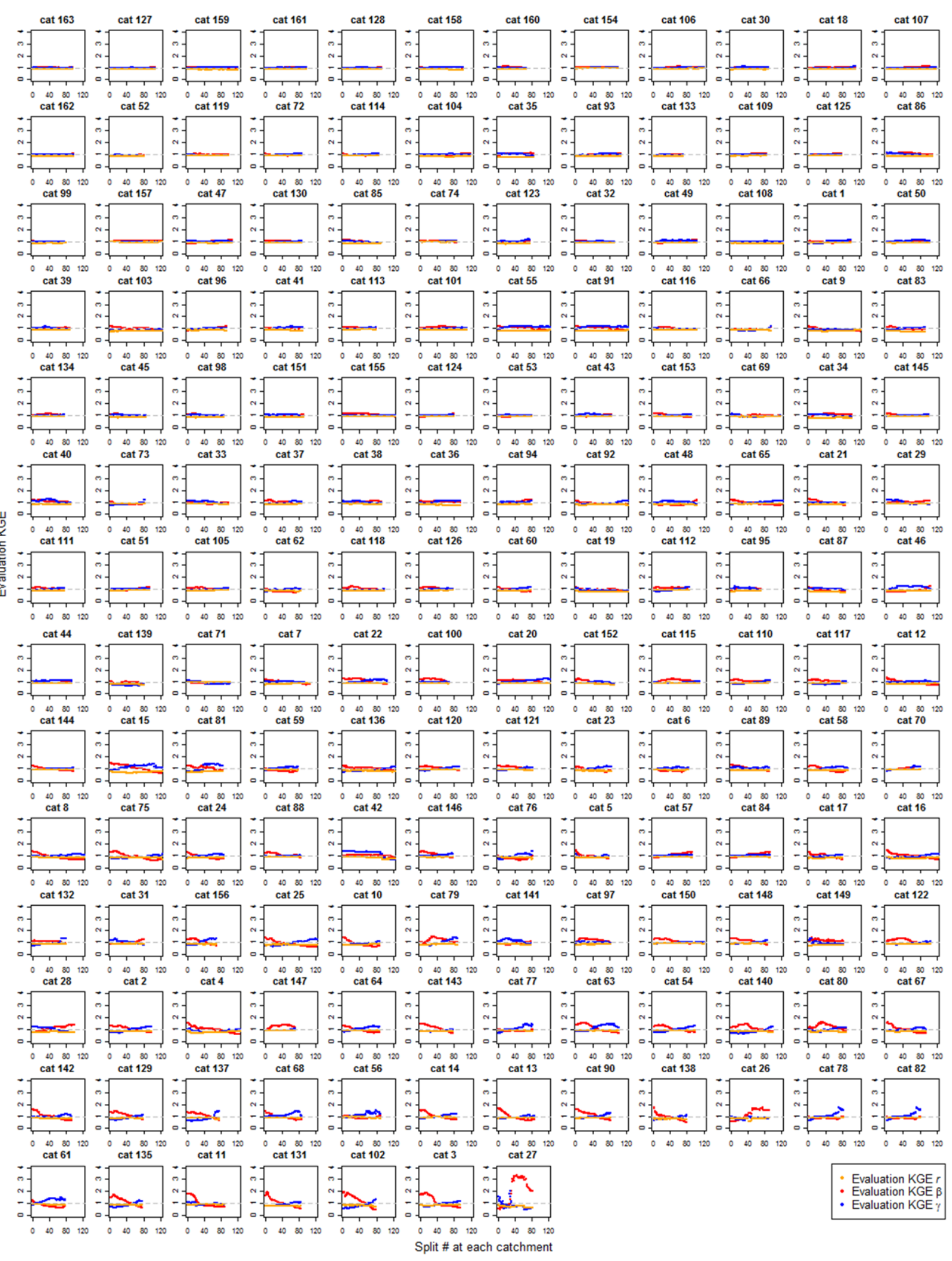


Figure 6. Evaluation KGE $r, \beta$ and $\gamma$ of GR4J, organized by catchment in each panel and plotted against the corresponding split number at each catchment. The horizontal grey dotted lines mark where KGE $r, \beta$ or $\gamma$ equals 1 (i.e. perfect match to observations). Catchments are sorted by increasing variance of overall evaluation KGE scores. 
[16] Confidential manuscript submitted to replace this text with name of AGU journal

[25] Interestingly, as also seen in Figure 6, at catchments where with higher variability of KGE, we generally observe a characteristic X-shaped pattern, indicating a 'compensation effect' between the evaluation $\beta$ and $Y$ (mean and variability) - which negatively correlated with each other. This is clearly seen in the last 20-30 catchments from the GR4J results, and to a lesser extent for the AWBM and CMD (Figures A1 and A2, respectively). This compensation effect is further illustrated in Figure 7 for two selected catchments with three models. At catchment $\# 163$, the variabilities of both evaluation $\beta$ and $\gamma$ are small across data splits. In contrast, at catchment \#102, both metrics are highly variable and show clear 'compensation' for all three models (although less distinct for CMD), such that overestimation of the mean $(\beta>1)$ tends to be associated with an underestimation of variability $(\Upsilon<1)$, and vice versa. 
Catchment 163
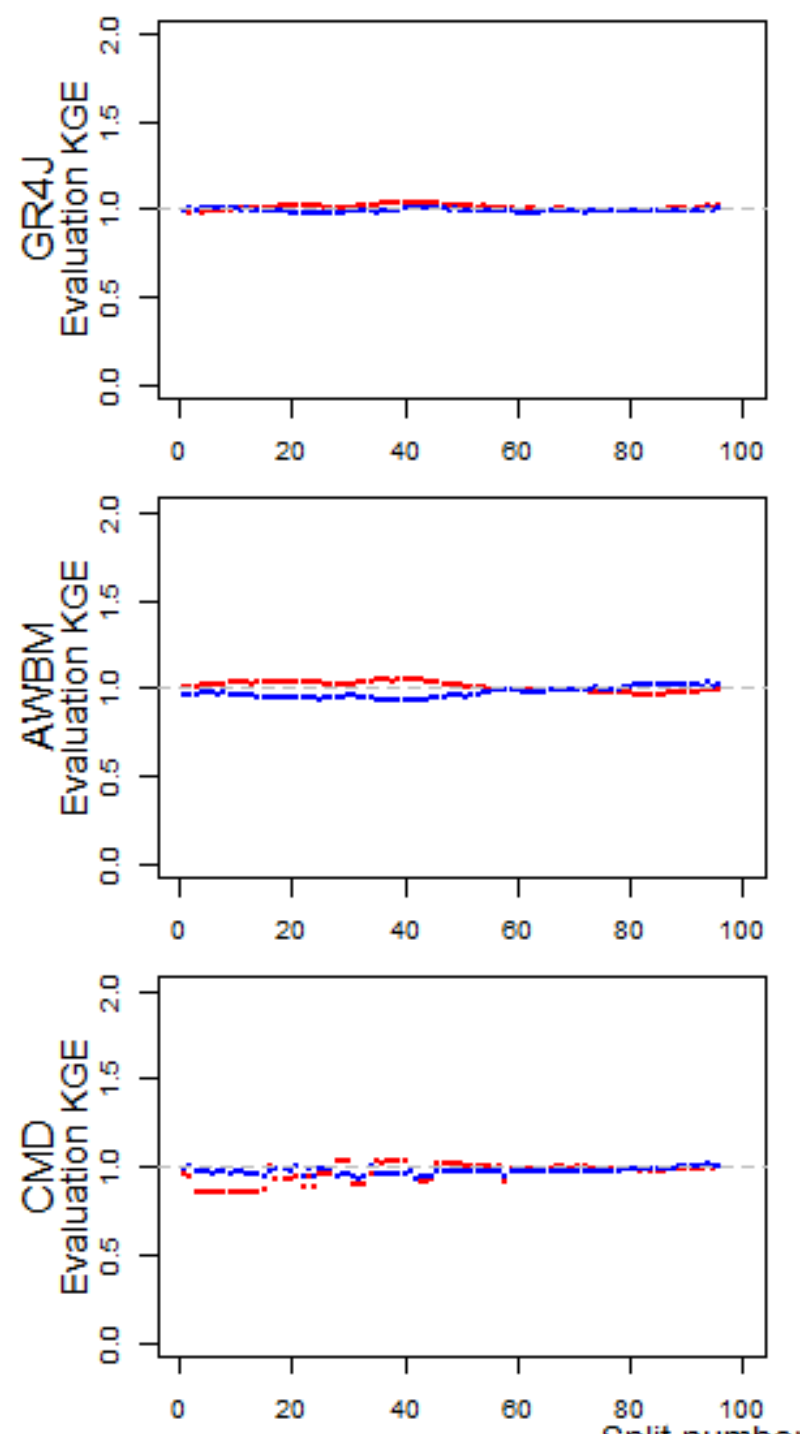

Split number at catchment
Catchment 102
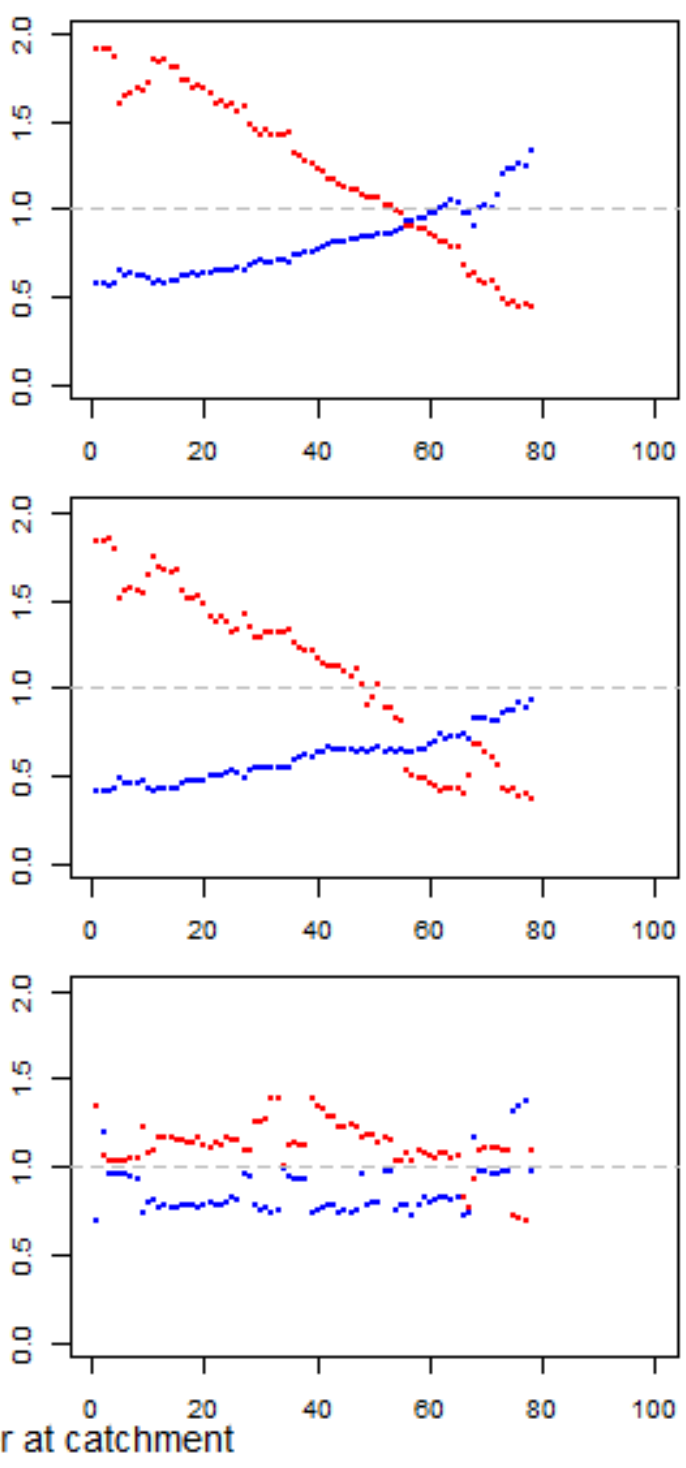

+ Evaluation KGE $\beta$ + Evaluation KGE $\eta$

Figure 7. Variation in evaluation KGE $\beta$ and $\gamma$ (y-axis) across various calibration/evaluation data splits (x-axis) at two catchments: left - catchment \#163 where variation in both metrices is small; right - catchment \#102, where both $\beta$ and $\gamma$ are highly variable across splits, and showing clear 'compensation effects'. Value on x- 
axis are ordered by the number of the calibration split at each catchment (i.e. by time; see Figure 3).

[26] Further to the compensation effects noted above, at most catchments that have strong compensation effects between evaluation $\beta$ and $\gamma$, the evaluation $\beta$ decreases while the evaluation $Y$ increases over time (i.e., along the $\mathrm{x}$-axes which correspond to the split number at each catchment) - as clearly illustrated with the GR4J results in Figure 6. This temporal trend in model performance suggests possible time-varying change of the rainfall-runoff relationship at these catchments. We further explore this with Figure 8, focusing on the long-term trends in the runoff at 20 catchments where the KGE displays widest range of variability for GR4J. At each catchment, values of annual average runoff are plotted as anomalies to the corresponding long-term average runoff. The majority of these catchments have experienced decreasing runoff over time; however, the corresponding rainfall anomalies do not show clear declining patterns, and so are not presented. These trends in runoff, in conjunction with the clear trends in the evaluation $\beta$ and $\gamma$ in Figure 6, suggest that CRR models that are calibrated to wetter periods tend to 1 ) over-estimate the mean runoff and 2) under-estimate the runoff variation, for dryer (evaluation) periods - as shown by the evaluation $\beta$ and $\gamma$ values for the first half of the splits, correspondingly. These results illustrate how changes in catchment behavior can affect the performance of CRR models, and are consistent with current findings on CRR model transferability [Bastola et al., 2011; Coron et al., 2012; Fowler et al., 2018] and are further discussed in Section 4. This is not further analyzed since time-varying behavior of catchments is out of scope for this study.

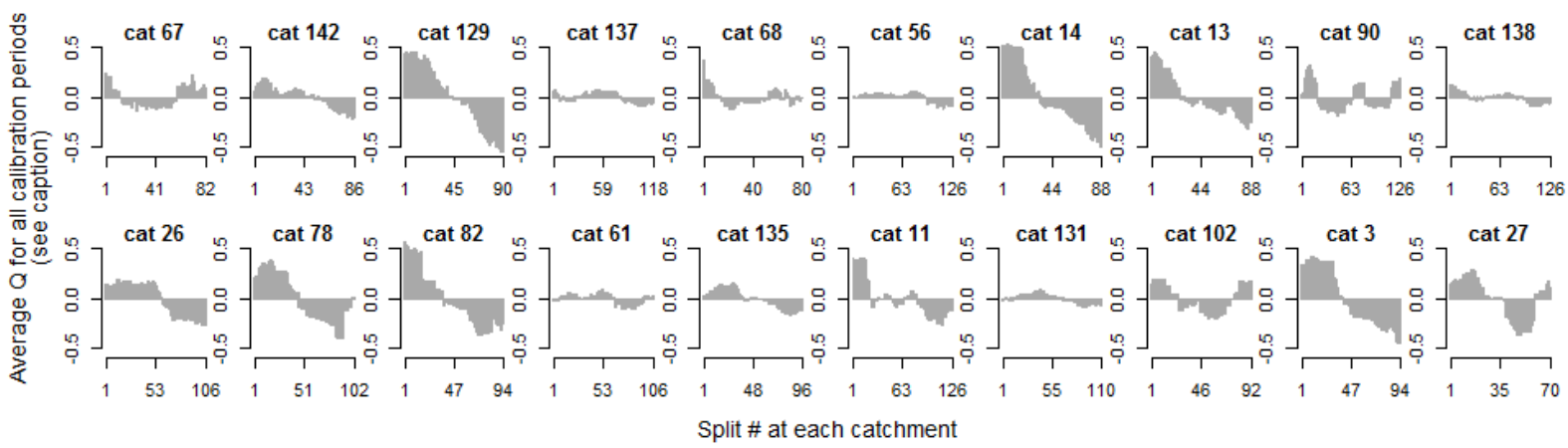

Figure 8. Averaged annual runoff of all calibration periods, at each of the 20 catchments which have the widest range of variation in evaluation KGE for GR4J. Note that all runoff values are shown as anomalies from the long-term annual average runoff.

[27] Regarding the robustness of CRR model performance, the compensation pattern seen in Figures 6 and 7 suggests that, for particular catchments, when evaluating across a large number 
of splits, it is for some reason very difficult for certain models to accurately simulate both the mean and variability ( $\beta$ and $\gamma$ respectively). The varying robustness of CRR models across catchments is likely related to the differences in hydro-climatic conditions at individual catchments. In Section 3.2 we investigate further how the long-term average conditions and the temporal variability of hydro-climatic conditions at a catchment may affect the robustness of evaluation performance for such catchments.

\subsection{Is there a relationship between CRR model robustness and the hydro-climatic} characteristics of catchments?

[28] Deeper investigation indicates that CRR model robustness (or lack thereof) seems to be closely related to catchment characteristics, with particularly strong effects being associated with the catchment aridity, rainfall-runoff ratio, runoff skewness, as well as temporal variability in baseflow contribution. Figure 9 illustrates how the performance robustness of the three models varies across the catchments with the hydro-climatic conditions, as represented by the 12 catchment characteristics introduced in Section 2.4. In these plots, the pairwise Spearman rank correlations are shown between the robustness of each model (represented by the standard deviation of KGE in the first three rows) and each of the 12 hydro-climatic characteristics at all catchments. Blue and red cells highlight negative and positive correlations, respectively, with pale colors indicating low/no correlations. In general, KGE standard deviation is most strongly negatively correlated with Mean_runoff, Mean_rainfall and Mean_RR_ratio; whereas the strongest positive correlations are observed with Mean_aridity, SD_aridity, SD_BFI and Skew_runoff. These patterns relating model robustness to catchment conditions are consistent across all models, although they illustrate weaker relationships for the robustness of CMD. 


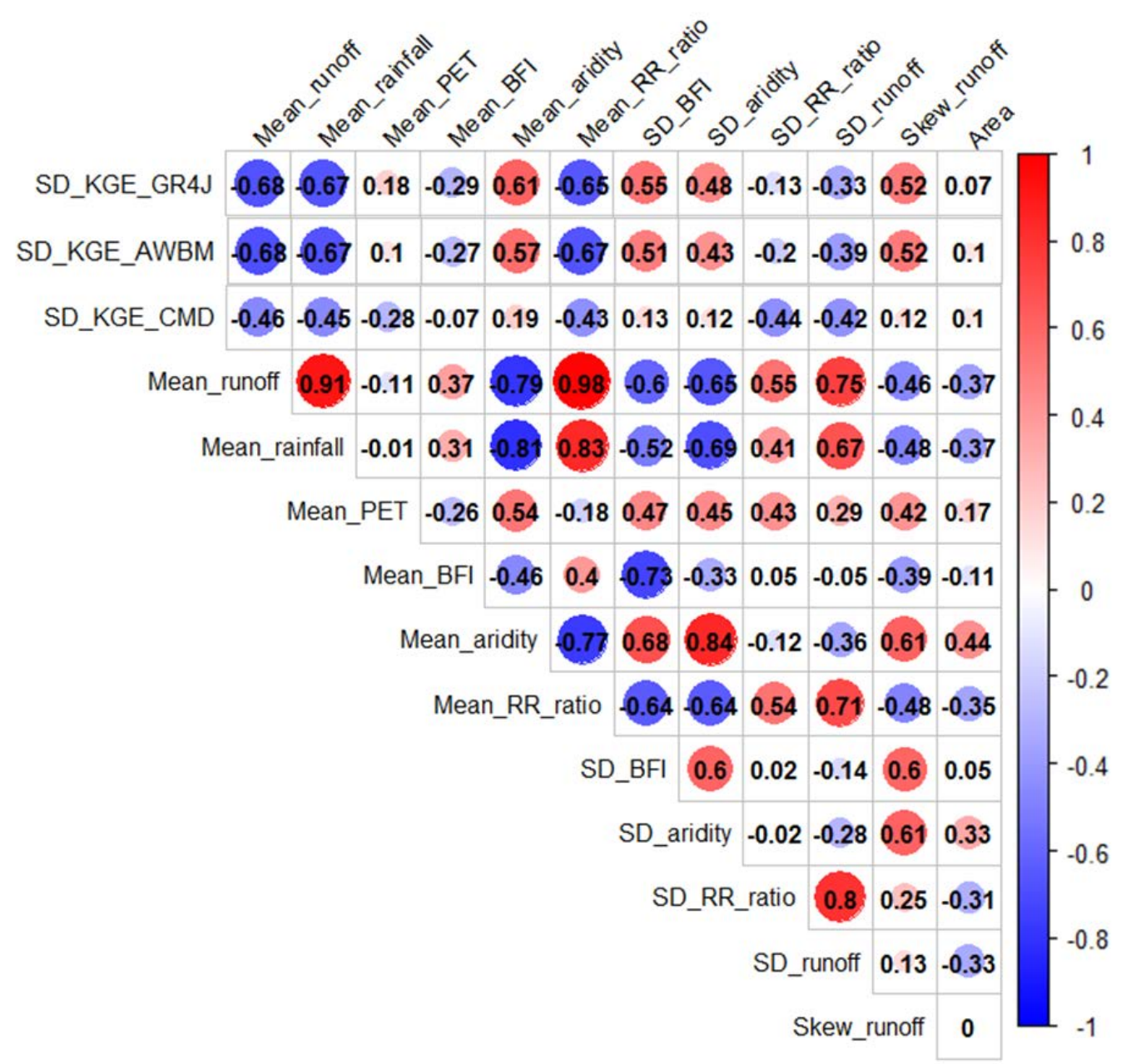

Figure 9. Spearman's rank correlations $(\mathrm{R})$ between model robustness (represented by standard deviation of KGE) and 12 catchment hydro-climatic characteristics, across all 163 catchments for GR4J, AWBM and CMD, in the first three rows, respectively. Correlations amongst the 12 hydro-climatic catchment characteristics (as defined in Table 1) are shown in rows 4 to 14 . Red and blue show positive and negative correlations, respectively, and pale colors show low/no correlations.

[29] These results indicate that lower performance robustness (i.e., higher KGE standard deviation) is better correlated to a number of catchment characteristics. While noting the high cross-correlations between them, the following four key characteristics seem to be representative of all correlated groups: 
[16] Confidential manuscript submitted to replace this text with name of AGU journal

1) Rainfall-runoff ratio is low, with low amounts in both annual rainfall and runoff (i.e. low Mean_RR_ratio, Mean_rainfall and Mean_runoff), which are all strongly positively correlated with Spearman's $\mathrm{R}$ values exceeding 0.83 ).

2) Aridity (PET relative to rainfall) is both high and variable over time (i.e. having high Mean_aridity and SD_aridity, which are strongly correlated with Spearman's R = 0.84); this is likely more closely related to low annual rainfall than to higher PET, as seen in the contrasting magnitudes of the correlations between performance robustness and Mean_rainfall and Mean_PET.

3) Runoff is highly positively skewed (i.e. high Skew_runoff), where extreme events tend to be rare and of high magnitude.

4) Proportion of baseflow contribution to runoff is highly variable (i.e. high $S D \_B F I$ ).

[30] Further analysis indicates some potential pathways via which these key catchment characteristics may influence model robustness. Figure 10 shows scatterplots of Mean_RR_ratio, Mean_aridity, Skew_runoff and SD_BFI, against the value of the store capacity that was obtained as a calibrated parameter from each model. For those plots, the store capacity value for each catchment was estimated by averaging corresponding parameter values across all calibration subperiods (see Section 2.3); each dot represents a catchment. In general, stronger correlations are seen between store capacity and the skewness of runoff. Specifically, as might be expected, higher runoff skewness is related to lower store capacity in each of the three models (although to a lesser extent in AWBM). Therefore, the robustness of CRR models to simulate the behaviors of these catchments seems to be somehow connected with the (smaller) sizes of the store capacities 'assigned' to them. We provide further discussion of this issue in Section 4. 


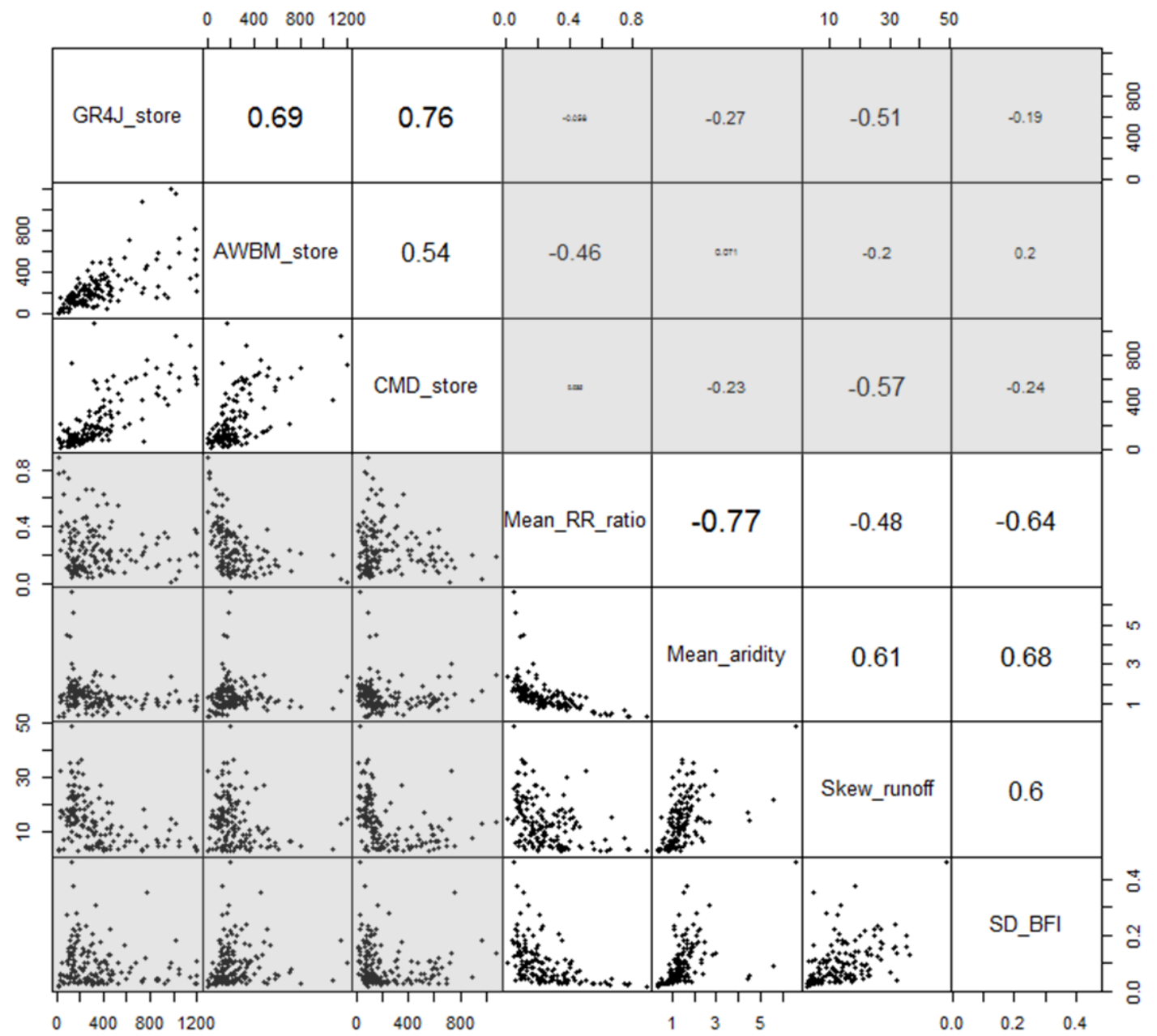

Figure 10. Relationships between Mean_RR_ratio, Mean_aridity, Skew_runoff and $S D \_B F I$ (as defined in Table 1), and the calibrated store capacity of each catchment from GR4J, AWBM and CMD (all in $\mathrm{mm}$ and averaged across all calibration subperiods, see Section 2.3). Shaded cells highlight correlations and scatter plots between each pair of catchment characteristic and calibrated store capacity. Each dot represents a catchment in the scatter plots. The pairwise Spearman's correlations (R) are shown in the top-right triangle, where larger text indicating greater magnitudes of correlations.

[31] To conclude this investigation, we summarize the overall pattern of the variation in CRR model robustness across differing catchment characteristics to provide guidelines for model 
[16] Confidential manuscript submitted to replace this text with name of AGU journal

selection before calibration is performed. To achieve this, we compare the robustness of the three CRR models for various groups of catchments that share similar conditions in of Mean_RR_ratio, Mean_aridity, Skew_runoff and SD_BFI (Figure 11). Specifically, we group all catchments by every $20^{\text {th }}$ quantile of values for each of the four catchment characteristics. For each KGE metric, we calculate the average of standard deviations for each of the five quantile groups, which is then plotted against the average of the corresponding catchment characteristic values for that group. Averaging across similar catchments enables us to eliminate some degree of 'noise' (variability across individual catchments), and to thereby better detect trends in the overall patterns of model robustness. 
[16] Confidential manuscript submitted to replace this text with name of AGU journal

KGE overall

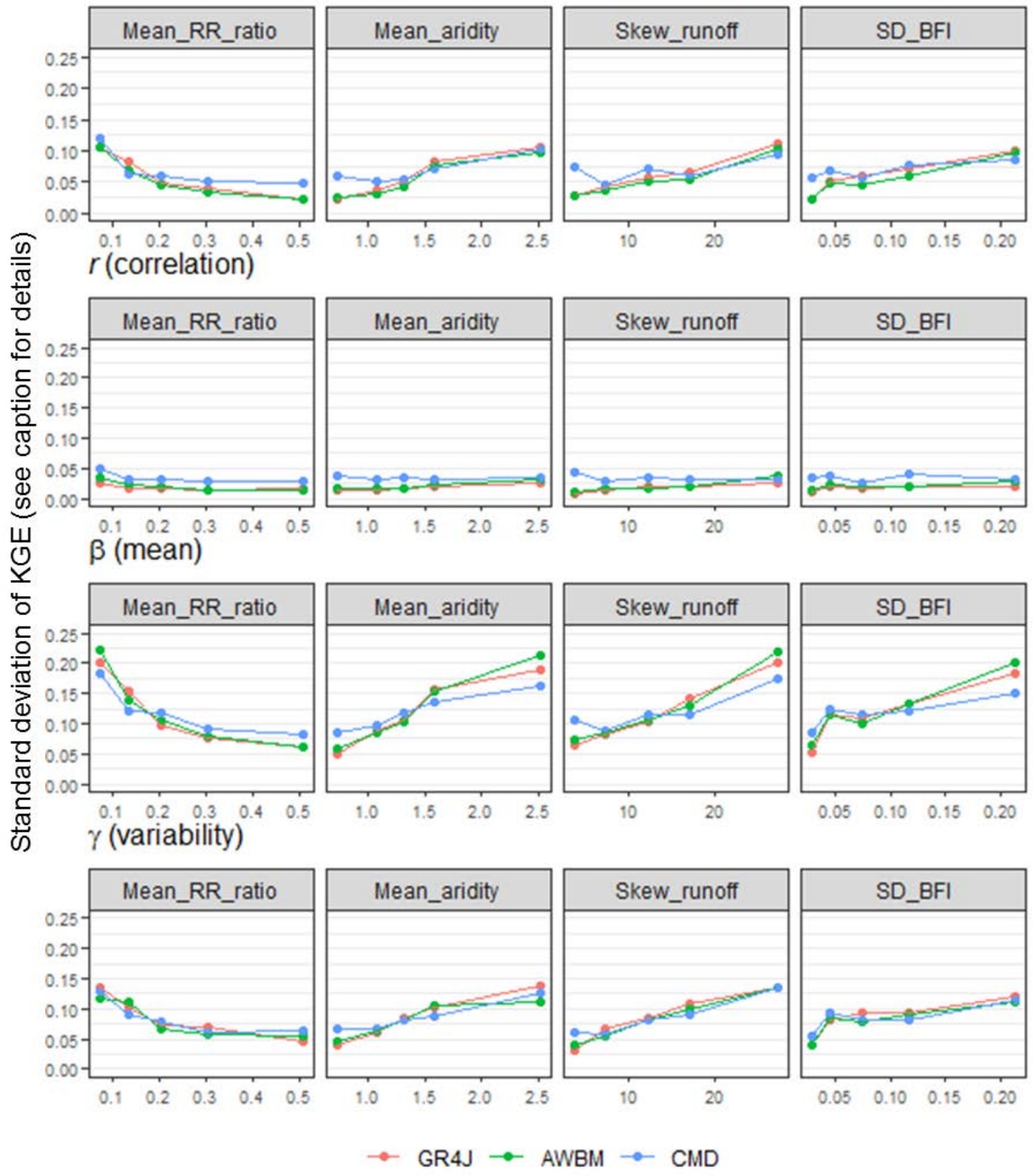

Figure 11. Robustness for each CRR model (characterized by the standard deviation of KGE), averaged across catchments of similar conditions in: Mean_RR_ratio,

This article is protected by copyright. All rights reserved. 
Mean_aridity, Skew_runoff and SD_BFI (in individual columns, see definitions in Table 1). Robustness is investigated in terms of KGE, $r, \beta$ and $\gamma$ in individual rows, and plotted against four key hydro-climatic conditions for all catchments. Y-axes show the average standard deviation of each KGE metric across catchments grouped by every $20^{\text {th }}$ quantile of the corresponding catchment characteristic (i.e. averaging over each of five groups of catchments). $\mathrm{X}$-axes show the three catchment characteristics in individual columns, averaged by every $20^{\text {th }}$ quantile of corresponding characteristic. Individual models are differentiated by color.

[32] Figure 11 shows that CMD generally exhibits the lowest robustness (highest KGE standard deviations), largely due to its low robustness in simulating the temporal structure of observed runoff (higher standard deviation of KGE $r$ ). GR4J is slightly less robust than AWBM when assessed by KGE overall; this is due to the combined effect of: (a) a generally higher robustness of GR4J in simulating runoff temporal structure (i.e. an overall lower standard deviation of KGE $r$ ); and (b) a generally lower robustness of GR4J in simulating both runoff mean and variability (i.e. higher standard deviations of KGE $\beta$ and $\gamma$ ), except for when aridity and runoff skewness are both low ( $<1$ and $<5$, respectively) and rainfall-runoff ratio is high ( $>$ $0.5)$.

[33] Overall, these results suggest the following model choices based on catchment characteristics:

1) AWBM is generally recommended when requiring higher robustness of KGE across a wide range of catchment conditions;

2) GR4J is recommended when the focus is high robustness in simulating the temporal structure of runoff;

3) GR4J is also recommended when aiming for high robustness in simulating the runoff mean and variability, at catchments with low aridity $(<1)$, low runoff skewness $(<5)$ and high rainfall-runoff ratio ( $>0.5)$.

[34] In summary, we observed substantial impacts of calibration-evaluation data split on CRR model performance. Across all models tested, performance robustness is higher in reproducing the temporal structure of runoff, compared with the mean and variability of runoff. Individual models seem to provide different levels of robustness depending on the specific performance metrics considered. Furthermore, the robustness of CRR model performance seems to be related to key hydro-climatic conditions. In general, it is more challenging to simultaneously achieve good performance during both calibration and evaluation periods when the catchments are drier (with high aridity and low rainfall-runoff ratio), where the contributions of baseflow are more temporally variable and where there are occasional extreme peak flow events. Section 4 presents more elaboration on key results and implications with respect to previous literature. 
[16] Confidential manuscript submitted to replace this text with name of AGU journal

\section{Discussions and Conclusions}

[35] Conceptual rainfall-runoff models are useful tools for a wide range of applications, from short-term forecasting to long-term water resources planning and management. However, several studies have suggested that these models tend to lack the ability to accurately simulate catchment responses for periods that are hydro-climatically different to those used for model calibration. Although this issue is well recognized, the extent of this problem across catchments, and its potential causes, are poorly understood.

[36] In this study, we have built upon a previous investigation of this issue that was conducted for data-based ANN rainfall-runoff models [Zheng et al., 2018]. An important difference between data-based and CRR models is that the former can typically be subjected to 'randomized sampling' when constructing the calibration/evaluation data splits, whereas the input-state-output form of CRR and other 'physically-based' models requires that timecontinuous data periods be used for model calibration. We applied a large-sample approach using 163 Australian catchments that span a range of hydro-climatic conditions, and investigated a large number of different possible calibration/evaluation splits at each catchment, to explore two research questions: 1) how robustly can CRR models perform with different instances of 50:50 calibration-evaluation data splits; 2) can any potential relationships be detected between model performance robustness and hydro-climatic characteristics of the catchments. Three popular CRR models (GR4J, AWBM and IHACRES-CMD) were used to test whether our findings are affected by differences in model structure.

[37] Overall, our results show that:

1) The use of different data splits can have a substantial impact on model performance, particularly on the ability of the model to accurately reproduce the mean and variability of runoff.

2) Lower robustness of CRR model performance tends to be associated with specific catchment characteristics, such as when runoff skewness and aridity are high, baseflow contribution is highly variable, and rainfall-runoff ratio is low.

3) Within the three CRR models investigated in this study (GR4J, AWBM and IHACRES_CMD), AWBM is generally more robust across various catchment conditions based on the overall KGE, whereas GR4J is more robust for simulating the temporal structure of runoff.

[38] Finding (2) indicates that the step involving partitioning of the data into calibration and evaluation periods requires particular care at drier catchments having generally low runoff and rare but extreme flow events. Further, runoff skewness seems to influence CRR model robustness, possibly due to the limited flexibility of process representation in the models. This 
is illustrated in Figure 10, where we see that when a model was calibrated to catchments with highly-skewed runoff distributions, low storage capacities (i.e. limited buffering capacity) were consistently estimated at these catchments for all three models.

[39] The relatively strong links found between model parameter values and catchment characteristics suggest that all three models have been reasonably well identified, which one might also expect due to their relatively simple structures [e.g. Shin et al., 2013]. As such, it seems unlikely that poor parameter identifiability is a key reason for the low model robustness observed in this study. Instead, low performance robustness at the drier catchments likely reflects model structural inadequacies, and in particular may be related to inflexibility of the smaller store capacity representations to accurately represent system behaviours during different hydro-climatic conditions. This highlights the need to investigate further the suitability of different CRR model structures for arid catchments with highly variable hydroclimate. One potential approach is to test how well these models represent the internal rainfall-runoff dynamics, such as quickflow, baseflow and actual evapotranspiration [Ferket et al., 2010; Guo et al., 2017; Li et al., 2015; Partington et al., 2013] at these catchments, across a range of hydro-climatic conditions.

[40] Our results also complement the previous model robustness study by Zheng et al. [2018] regarding identifying catchments that are likely associated with low rainfall-runoff model robustness. Our finding that runoff skewness is a key factor affecting CRR model robustness is consistent with that by Zheng et al. [2018] for data-based ANN rainfall-runoff models. However, the two studies illustrate contrasting pathways through which runoff skewness can affect model robustness. For the data-driven ANN models used in Zheng et al. [2018], the impact of highly skewed runoff can be explained purely from a statistical perspective, where the presence of more extreme data points makes it more difficult to have a similar distribution of representative data in both calibration and evaluation datasets. In contrast, the use of CRR models in this study suggest that the sensitivity of model performance to the choice of data periods can be a result of different model conceptualizations, which arise under contrasting hydro-climatic conditions between different calibration periods.

[41] Comparing these two studies, it seems that runoff skewness can affect model robustness in different ways that are related to the varying roles of process representation and input data on different types of rainfall-runoff models [as discussed in Mount et al., 2016]. This comparison also suggests potential approaches to improving robustness for different types of rainfall-runoff models. Zheng et al. [2018] showed that the impact of runoff skewness on ANN model robustness can be minimized by using appropriate data splitting methods, but this result does not readily transfer to CRR models due to their more rigid data structure requirement (temporally), as discussed previously. Considering the limitations of fixed model structure on 
model robustness as shown in this study (Figure 10), we suggest a potential approach to improving robustness of CRR models via more flexible model structural representations that can accommodate a wider range of hydro-climatic conditions.

[42] The temporal transferability of CRR models is a topic that is closely linked to model robustness, while being better understood. An increasing number of studies have attributed low model transferability to time-variations in catchment conditions [e.g. Bastola et al., 2011; Coron et al., 2012; Fowler et al., 2018], which largely focused on the contrasting dry/wet conditions between calibration and evaluation periods. Consistent results are also seen in Section 3.1 in this study, where a common over-estimation is noted for mean runoff when CRR models are calibrated to periods wetter than the evaluation period.

[43] Our analyses in Section 3.2, while addressing the issue of model robustness associated with transferability in time, can also be viewed as a more general investigation of the range of CRR performance variation by catchment, and thus pointing towards potential causes for unstable model performance. For example, within the previous studies that attempted to explain low transferability of CRR models [e.g. Bastola et al., 2011; Coron et al., 2012; Fowler et al., 2018], the association between unstable CRR model performance and high runoff skewness has not been reported. One possible explanation is that if runoff skewness is consistently high at a catchment throughout the entire dataset, it would not be easily noted from a comparison between calibration and evaluation datasets - which has been the focus of previous transferability investigations; in contrast, focusing on characteristics of individual catchments (as illustrated in this study) allowed us to study the influence of a wider range of catchment conditions. This illustrates the potential value of performing comprehensive investigations focusing on model robustness instead of transferability.

[44] In conclusion, the large-sample approach is increasingly being used to support model development, to estimate and/or test model parameters, and to conduct large-scale impact assessments [Gupta et al., 2014]. This is the first large-sample study to focus specifically on the performance robustness of CRR models and its key controls. In the spirit of previous largesample works for model benchmarking [e.g. Newman et al., 2017; Wu et al., 2013], our results can be considered a useful benchmark of expected variability in model performance when different calibration and evaluation splits are used at a specific catchment. It is worth noting that the impacts of record lengths for calibration/evaluation periods remains as an important outstanding question. Coron et al. [2012] discussed the key considerations in choosing calibration periods when applying the split-sample approach to test model transferability, which is essentially a choice between maximizing information content (i.e. hydrologic variability) either within each split or across different splits. Specifically, the length of calibration data should be sufficient to become informative for accurate parameter estimation. At the same time, 
longer calibration periods may also 1) lead to less contrast between periods; and 2) reduce the number of independent calibration periods and thus act counter to the purpose of split-sample calibration. We acknowledge that the 50:50 split we used in this study generally led to longer calibration periods (>15 years) than those used in typical CRR model calibrations, and the impacts of shorter calibration periods on our results need to be further understood. However, it should be noted that the length of the calibration period is less important than the variability in the events/patterns (i.e. the information content) contained in the calibration data. For example, shorter calibration periods are required for catchments with similar events/patterns to enable all relevant information to be captured during the calibration process. Consequently, a detailed study of the impact of which percentage of the available data are used for calibration would also need to consider the length of the available data relative to the degree of variability in the types of patterns/events contained in the data.

[45] In addition, this study has used KGE as the only objective function for model calibration. Some recent studies suggested least-square based evaluation metrics such as the KGE and the Nash-Sutcliffe Efficiency (NSE) [Nash \& Sutcliffe, 1970] can be limited in identifying rainfallrunoff models that are robust under changing catchment flow regimes, since these metrics commonly favour parameter sets that provide better match to high-flow conditions [Fowler et al., 2018; Fowler et al., 2016; Krause et al., 2005]. Therefore, future studies should assess the impact of alternative metrics (such as KGE on transformed streamflow, as suggested in Fowler et al. [2018]) on the findings of this work.

[46] While the choice of objective function and which percentage of the available data to use for calibration and evaluation could have some impact on the specific recommendations of this study, the underlying principles observed are likely to remain unchanged, as they are underpinned by explanations of underlying catchment processes. Consequently, this study provides valuable insights into the potential impact of the choice of which subset of the available data to use for calibration and which subset to use for evaluation on model performance for catchments with different characteristics, as well as high-level guidance on how any potential issues might be addressed. 


\section{Acknowledgments, Samples, and Data}

Professor Zheng is funded by the National Natural Science Foundation of China (Grant No. 51922096). Professor Gupta acknowledges partial support from the Australian Research Council through the Centre of Excellence for Climate System Science (grant CE110001028). The historical streamflow data for all study catchments are available from the Australian Bureau of Meteorology (BoM) Hydrological Reference Stations, available from http://www.bom.gov.au/water/hrs/. The historical rainfall and PET data were from the BoM Australian Water Availability Project (AWAP), at http://www.csiro.au/awap/. The authors would also like to thank Ms. Jie Jian for her great help on data input, and Mr. Luis de la Fuente for his kind support to investigate how model robustness might be related to catchment characteristics.

\section{References}

[47] Andrews, F., \& Guillaume, J. (2013). hydromad: Hydrological Model Assessment and Development. R package version 0.9-18. Retrieved 15/07/2014 http://hydromad.catchment.org/

[48] Andrews, F. T., Croke, B. F. W., \& Jakeman, A. J. (2011). An open software environment for hydrological model assessment and development. Environmental Modelling \& Software, 26(10), 11711185.

[49] Bastola, S., Murphy, C., \& Sweeney, J. (2011). Evaluation of the transferability of hydrological model parameters for simulations under changed climatic conditions. Hydrol. Earth Syst. Sci. Discuss., 2011, 5891-5915. doi:10.5194/hessd-8-5891-2011

[50] Beven, K. (1989). Changing ideas in hydrology - The case of physically-based models. Journal of Hydrology, 105(1), 157-172. doi:https://doi.org/10.1016/0022-1694(89)90101-7

[51] Beven, K. J. (2011). Rainfall-Runoff Modelling: The Primer: Wiley.

[52] Boughton, W. (2004). The Australian water balance model. Environmental Modelling \& Software, 19(10), 943-956.

[53] Boughton, W., \& Droop, O. (2003). Continuous simulation for design flood estimation-a review. Environmental Modelling \& Software, 18(4), 309-318. doi:http://dx.doi.org/10.1016/S13648152(03)00004-5

[54] Boughton, W. C. (1993). A hydrograph-based model for estimating the water yield of ungauged catchments. Paper presented at the Hydrology and Water Resources Conference, Institution of Engineers, Australia.

[55] Boughton, W. C., \& Carroll, D. G. (1993). A simple combined water balance/flood hydrograph model. Paper presented at the Hydrology and Water Resources Conference, Institution of Engineers, Australia.

[56] Broderick, C., Matthews, T., Wilby, R. L., Bastola, S., \& Murphy, C. (2016). Transferability of hydrological models and ensemble averaging methods between contrasting climatic periods. Water Resources Research, 52(10), 8343-8373. doi:doi:10.1002/2016WR018850 
[16] Confidential manuscript submitted to replace this text with name of AGU journal

[57] Coron, L., Andréassian, V., Perrin, C., Bourqui, M., \& Hendrickx, F. (2014). On the lack of robustness of hydrologic models regarding water balance simulation: a diagnostic approach applied to three models of increasing complexity on 20 mountainous catchments. Hydrol. Earth Syst. Sci., 18(2), 727-746. doi:10.5194/hess-18-727-2014

[58] Coron, L., Andréassian, V., Perrin, C., Lerat, J., Vaze, J., Bourqui, M., et al. (2012). Crash testing hydrological models in contrasted climate conditions: An experiment on 216 Australian catchments. Water Resources Research, 48(5), n/a-n/a. doi:10.1029/2011WR011721

[59] Croke, B. F. W., \& Jakeman, A. J. (2004). A catchment moisture deficit module for the IHACRES rainfall-runoff model. Environmental Modelling and Software with Environment Data News, 19(1), 1-5. doi:10.1016/j.envsoft.2003.09.001

[60] Ferket, B. V. A., Samain, B., \& Pauwels, V. R. N. (2010). Internal validation of conceptual rainfallrunoff models using baseflow separation. Journal of Hydrology, 381(1), 158-173. doi:https://doi.org/10.1016/j.jhydrol.2009.11.038

[61] Fowler, K., Peel, M., Western, A., \& Zhang, L. (2018). Improved Rainfall-Runoff Calibration for Drying Climate: Choice of Objective Function. Water Resources Research, 54(5), 3392-3408. doi:10.1029/2017wr022466

[62] Fowler, K. J. A., Peel, M. C., Western, A. W., Zhang, L., \& Peterson, T. J. (2016). Simulating runoff under changing climatic conditions: Revisiting an apparent deficiency of conceptual rainfall-runoff models. Water Resources Research, 52(3), 1820-1846. doi:10.1002/2015WR018068

[63] Galelli, S., Humphrey, G. B., Maier, H. R., Castelletti, A., Dandy, G. C., \& Gibbs, M. S. (2014). An evaluation framework for input variable selection algorithms for environmental data-driven models. Environmental Modelling \& Software, 62(0), 33-51. doi:http://dx.doi.org/10.1016/j.envsoft.2014.08.015

[64] Gibbs, M., McInerney, D., Humphrey, G., Thyer, M., Maier, H., Dandy, G., et al. (2018). State updating and calibration period selection to improve dynamic monthly streamflow forecasts for an environmental flow management application.

[65] Gibbs, M. S., Maier, H. R., \& Dandy, G. C. (2012). A generic framework for regression regionalization in ungauged catchments. Environmental Modelling \& Software, 27-28, 1-14. doi:http://dx.doi.org/10.1016/j.envsoft.2011.10.006

[66] Gudmundsson, L., Wagener, T., Tallaksen, L. M., \& Engeland, K. (2012). Evaluation of nine large-scale hydrological models with respect to the seasonal runoff climatology in Europe. Water Resources Research, 48(11). doi:10.1029/2011wr010911

[67] Guo, D., Westra, S., \& Maier, H. R. (2017). Impact of evapotranspiration process representation on runoff projections from conceptual rainfall-runoff models. Water Resources Research, 53. doi:10.1002/2016WR019627

[68] Gupta, H. V., Kling, H., Yilmaz, K. K., \& Martinez, G. F. (2009). Decomposition of the mean squared error and NSE performance criteria: Implications for improving hydrological modelling. Journal of Hydrology, 377(1), 80-91. doi:https://doi.org/10.1016/j.jhydrol.2009.08.003

[69] Gupta, H. V., Perrin, C., Blöschl, G., Montanari, A., Kumar, R., Clark, M., et al. (2014). Large-sample hydrology: a need to balance depth with breadth. Hydrol. Earth Syst. Sci., 18(2), 463-477. doi:10.5194/hess-18-463-2014 
[16] Confidential manuscript submitted to replace this text with name of AGU journal

[70] Gupta, V. K., \& Sorooshian, S. (1985). The relationship between data and the precision of parameter estimates of hydrologic models. Journal of Hydrology, 81(1), 57-77. doi:https://doi.org/10.1016/00221694(85)90167-2

[71] Hartmann, G., \& Bárdossy, A. (2005). Investigation of the transferability of hydrological models and a method to improve model calibration. Advances in Geosciences, 5, 83-87.

[72] Jones, R. N., Chiew, F. H. S., Boughton, W. C., \& Zhang, L. (2006). Estimating the sensitivity of mean annual runoff to climate change using selected hydrological models. Advances in Water Resources, 29(10), 1419-1429. doi:http://dx.doi.org/10.1016/j.advwatres.2005.11.001

[73] Kay, A. L., Jones, R. G., \& Reynard, N. S. (2006). RCM rainfall for UK flood frequency estimation. II. Climate change results. Journal of Hydrology, 318(1-4), 163-172. doi:http://doi.org/10.1016/j.jhydrol.2005.06.013

[74] KlemeŠ, V. (1986). Operational testing of hydrological simulation models. Hydrological Sciences Journal, 31(1), 13-24. doi:10.1080/02626668609491024

[75] Kling, H., Fuchs, M., \& Paulin, M. (2012). Runoff conditions in the upper Danube basin under an ensemble of climate change scenarios. Journal of Hydrology, 424, 264-277.

[76] Krause, P., Boyle, D. P., \& Bäse, F. (2005). Comparison of different efficiency criteria for hydrological model assessment. Advances in Geosciences, 5, 89-97.

[77] Li, C., Zhang, L., Wang, H., Zhang, Y., Yu, F., \& Yan, D. (2012). The transferability of hydrological models under nonstationary climatic conditions. Hydrology and Earth System Sciences, 16(4), 1239-1254.

[78] Li, L., Lambert, M. F., Maier, H. R., Partington, D., \& Simmons, C. T. (2015). Assessment of the internal dynamics of the Australian Water Balance Model under different calibration regimes. Environmental Modelling \& Software, 66, 57-68. doi:https://doi.org/10.1016/j.envsoft.2014.12.015

[79] Maier, H. R., Jain, A., Dandy, G. C., \& Sudheer, K. P. (2010). Methods used for the development of neural networks for the prediction of water resource variables in river systems: Current status and future $\begin{array}{lllll}\text { directions. } \quad \text { Environmental } & \text { Modelling } \& \text { Software, }\end{array}$ doi:https://doi.org/10.1016/j.envsoft.2010.02.003

[80] Maier, H. R., Razavi, S., Kapelan, Z., Matott, L. S., Kasprzyk, J., \& Tolson, B. A. (2019). Introductory overview: Optimization using evolutionary algorithms and other metaheuristics. Environmental Modelling \& Software, 114, 195-213. doi:https://doi.org/10.1016/j.envsoft.2018.11.018

[81] Merz, R., Parajka, J., \& Blöschl, G. (2011). Time stability of catchment model parameters: Implications for climate impact analyses. Water Resources Research, 47(2), n/a-n/a. doi:10.1029/2010WR009505

[82] Mount, N. J., Maier, H. R., Toth, E., Elshorbagy, A., Solomatine, D., Chang, F. J., et al. (2016). Datadriven modelling approaches for socio-hydrology: opportunities and challenges within the Panta Rhei Science Plan. Hydrological Sciences Journal, 61(7), 1192-1208. doi:10.1080/02626667.2016.1159683

[83] Murphy, C., Fealy, R., Charlton, R., \& Sweeney, J. (2006). The Reliability of an 'Off-the-Shelf' Conceptual Rainfall Runoff Model for Use in Climate Impact Assessment: Uncertainty Quantification Using Latin Hypercube Sampling. Area, 38(1), 65-78.

[84] Najafi, M. R., \& Moradkhani, H. (2015). Multi-model ensemble analysis of runoff extremes for climate change impact assessments. Journal of Hydrology, 525, 352-361. doi:http://dx.doi.org/10.1016/j.jhydrol.2015.03.045 
[16] Confidential manuscript submitted to replace this text with name of AGU journal

[85] Nash, J. E., \& Sutcliffe, J. V. (1970). River flow forecasting through conceptual models part I - A discussion of principles. Journal of Hydrology, 10(3), 282-290. doi:https://doi.org/10.1016/0022$\underline{1694(70) 90255-6}$

[86] Newman, A. J., Mizukami, N., Clark, M. P., Wood, A. W., Nijssen, B., \& Nearing, G. (2017). Benchmarking of a Physically Based Hydrologic Model. Journal of Hydrometeorology, 18(8), 2215-2225. doi:10.1175/jhm-d-16-0284.1

[87] Partington, D., Brunner, P., Frei, S., Simmons, C. T., Werner, A. D., Therrien, R., et al. (2013). Interpreting streamflow generation mechanisms from integrated surface-subsurface flow models of a riparian wetland and catchment. Water Resources Research, 49(9), 5501-5519. doi:10.1002/wrcr.20405

[88] Perrin, C., Michel, C., \& Andréassian, V. (2003). Improvement of a parsimonious model for streamflow simulation. Journal of Hydrology, 279(1), 275-289.

[89] Raupach, M., Briggs, P., Haverd, V., King, E., Paget, M., \& Trudinger, C. (2009). Australian water availability project (AWAP): CSIRO marine and atmospheric research component: final report for phase 3. Retrieved from

[90] Raupach, M., Briggs, P., Haverd, V., King, E., Paget, M., \& Trudinger, C. (2012). Australian Water Availability Project. CSIRO Marine and Atmospheric Research, Canberra, Australia. Retrieved from: http://www.csiro.au/awap

[91] Shin, M.-J., Guillaume, J. H. A., Croke, B. F. W., \& Jakeman, A. J. (2013). Addressing ten questions about conceptual rainfall-runoff models with global sensitivity analyses in R. Journal of Hydrology, 503, 135-152. doi:https://doi.org/10.1016/j.jhydrol.2013.08.047

[92] Shin, M.-J., Guillaume, J. H. A., Croke, B. F. W., \& Jakeman, A. J. (2015). A review of foundational methods for checking the structural identifiability of models: Results for rainfall-runoff. Journal of Hydrology, 520, 1-16. doi:https://doi.org/10.1016/j.jhydrol.2014.11.040

[93] Sorooshian, S., Gupta, V. K., \& Fulton, J. L. (1983). Evaluation of Maximum Likelihood Parameter estimation techniques for conceptual rainfall-runoff models: Influence of calibration data variability and length on model credibility. Water Resources Research, 19(1), 251-259. doi:10.1029/WR019i001p00251

[94] Stern, H., De Hoedt, G., \& Ernst, J. (2000). Objective classification of Australian climates. Australian Meteorological Magazine, 49(2), 87-96.

[95] Vaze, J., Post, D. A., Chiew, F. H. S., Perraud, J. M., Viney, N. R., \& Teng, J. (2010). Climate nonstationarity - Validity of calibrated rainfall-runoff models for use in climate change studies. Journal of Hydrology, 394(3-4), 447-457. doi:http://dx.doi.org/10.1016/j.jhydrol.2010.09.018

[96] Vaze, J., \& Teng, J. (2011). Future climate and runoff projections across New South Wales, Australia: results and practical applications. Hydrological Processes, 25(1), 18-35. doi:10.1002/hyp.7812

[97] Wagener, T., McIntyre, N., Lees, M. J., Wheater, H. S., \& Gupta, H. V. (2003). Towards reduced uncertainty in conceptual rainfall-runoff modelling: dynamic identifiability analysis. Hydrological Processes, 17(2), 455-476. doi:10.1002/hyp.1135

[98] Wu, W., May, R. J., Maier, H. R., \& Dandy, G. C. (2013). A benchmarking approach for comparing data splitting methods for modeling water resources parameters using artificial neural networks. Water Resources Research, 49(11), 7598-7614. 
[16] Confidential manuscript submitted to replace this text with name of AGU journal

[99] Yapo, P. O., Gupta, H. V., \& Sorooshian, S. (1996). Automatic calibration of conceptual rainfall-runoff models: sensitivity to calibration data. Journal of Hydrology, 181(1), 23-48. doi:https://doi.org/10.1016/0022-1694(95)02918-4

[100] Zheng, F., Maier, H. R., Wu, W., Dandy, G. C., Gupta, H. V., \& Zhang, T. (2018). On Lack of Robustness in Hydrological Model Development Due to Absence of Guidelines for Selecting Calibration and Evaluation Data: Demonstration for Data - Driven Models. Water Resources Research, 54(2), 10131030. doi:doi:10.1002/2017WR021470

[101] Zheng, F., Westra, S., \& Leonard, M. (2015). Opposing local precipitation extremes. Nature Climate Change, 5, 389. doi:10.1038/nclimate2579

[102] https://www.nature.com/articles/nclimate2579\#supplementary-information 


\section{Appendix}

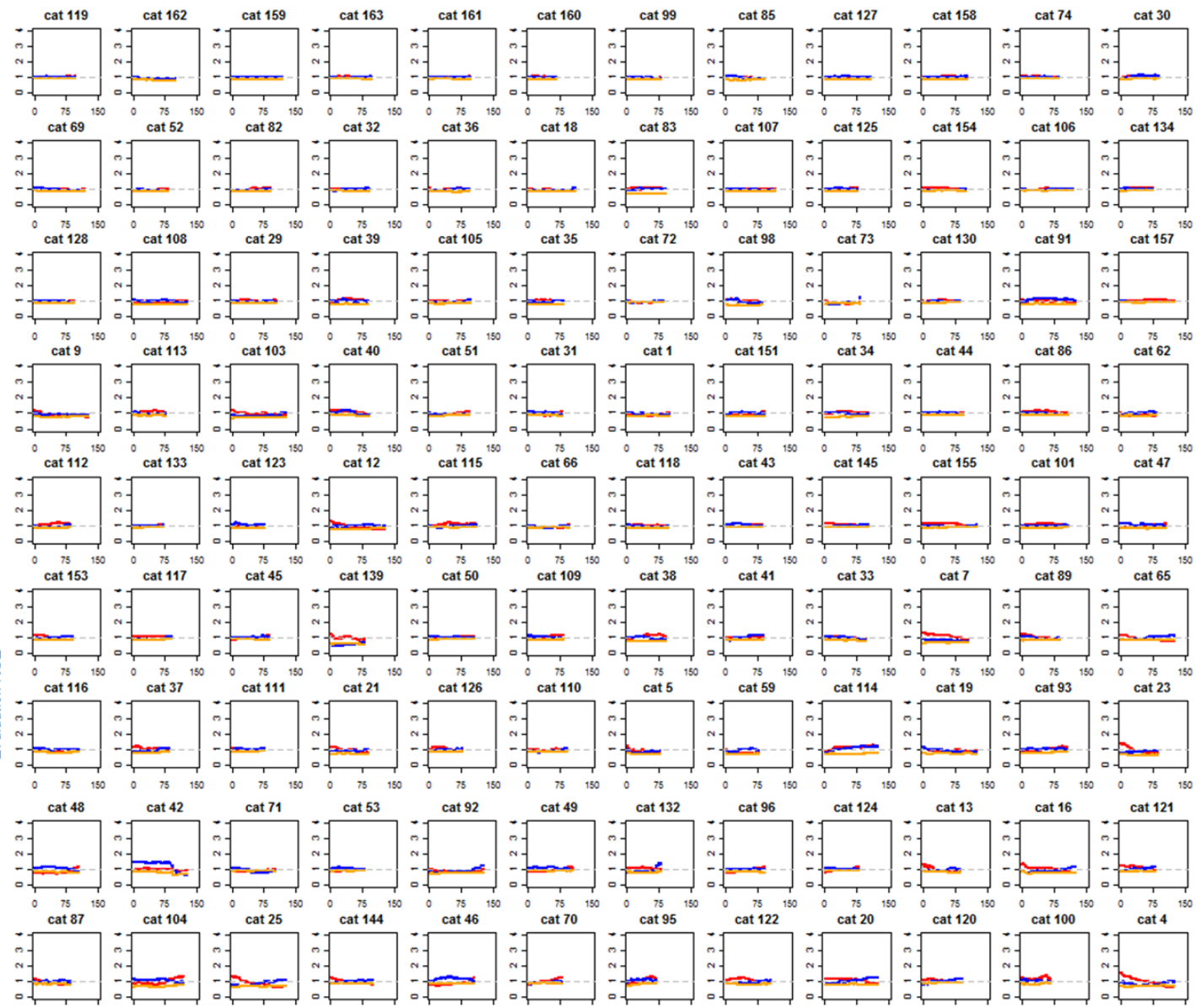

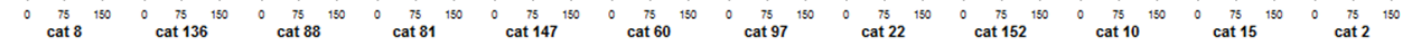

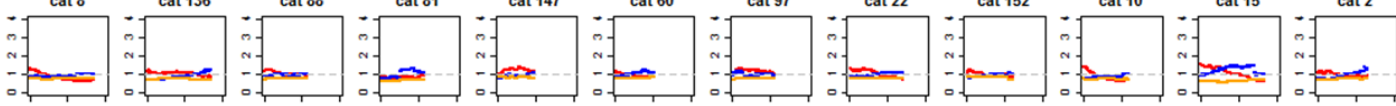

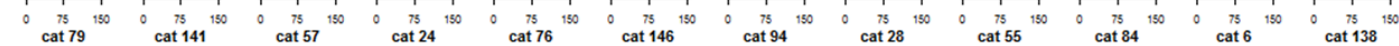

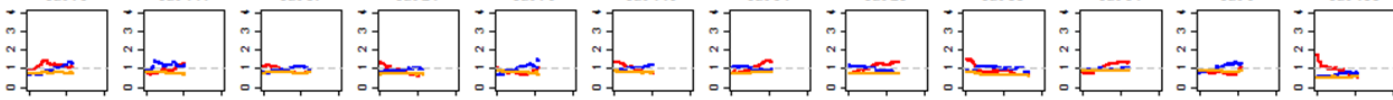

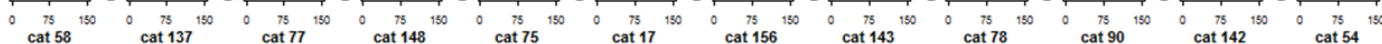

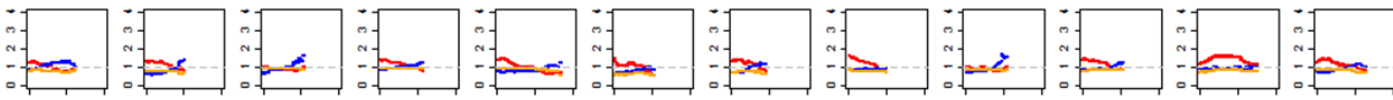

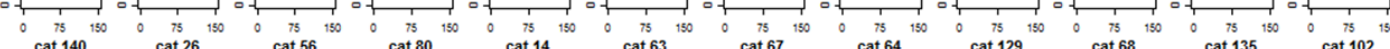

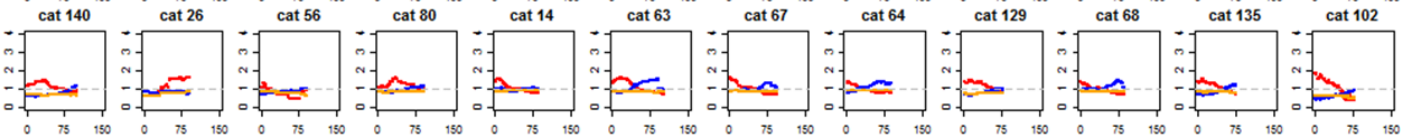

$\begin{array}{lllllllllllllllllllll}0 & 75 & 150 & 0 & 75 & 150 & 0 & 75 & 150 & 0 & 75 & 150 & 0 & 75 & 150 & 0 & 75 & 150 & 0 & 75 & 150\end{array}$

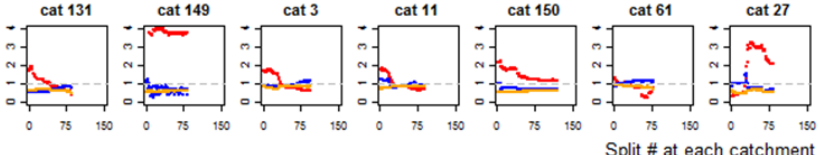

: Evaluation KGE $r$ - Evaluation KGE? 
[16] Confidential manuscript submitted to replace this text with name of AGU journal

Figure A1. Evaluation KGE $r, \beta$ and $\Upsilon$ of AWBM, organized by catchment in each panel and plotted against the corresponding split number at each catchment. The horizontal grey dotted lines mark where KGE $r, \beta$ or $\gamma$ equals 1 (i.e. perfect match to observations). Catchments are sorted by increasing variance of overall evaluation KGE scores. 

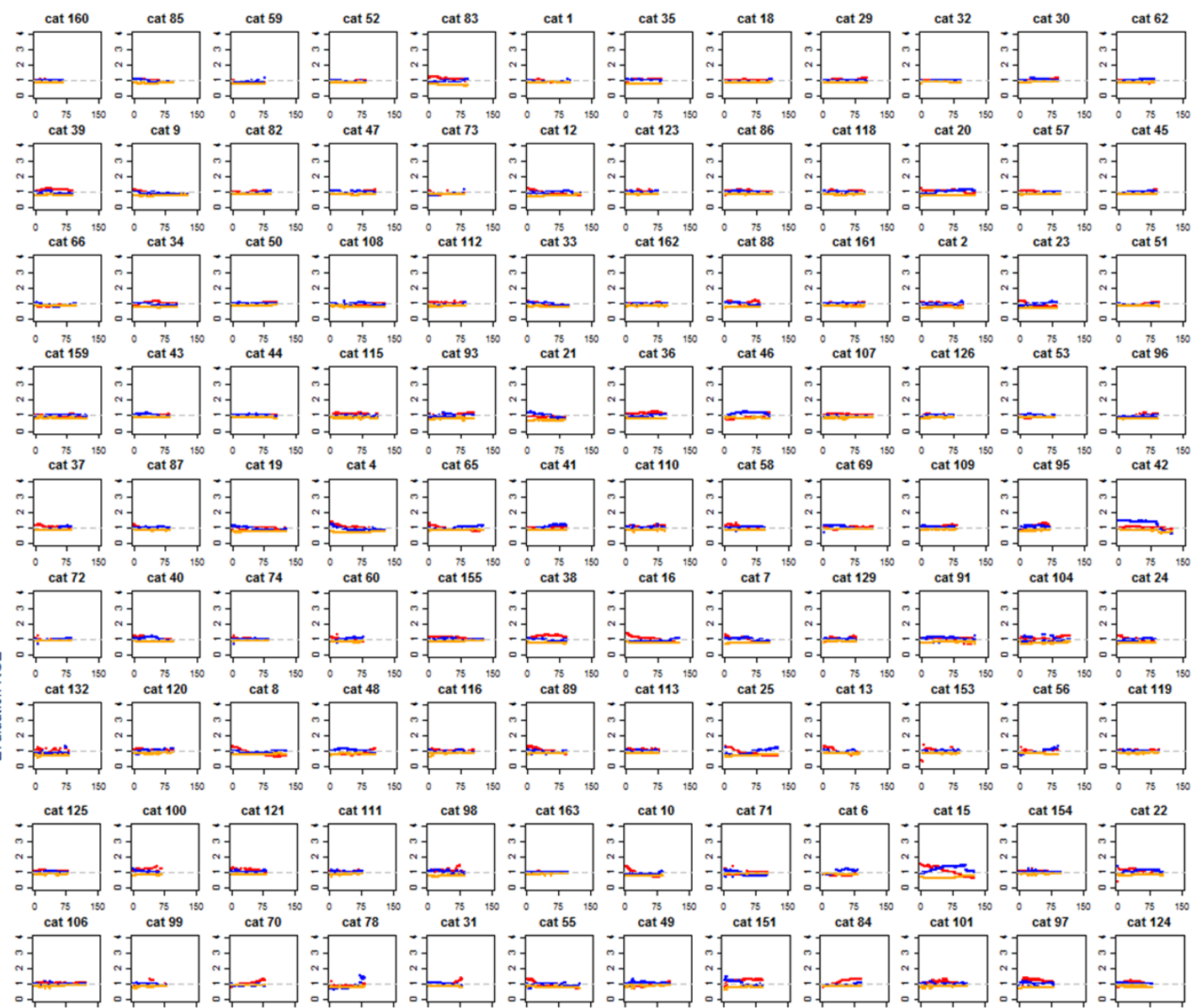

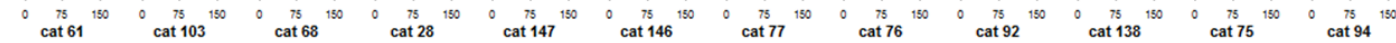

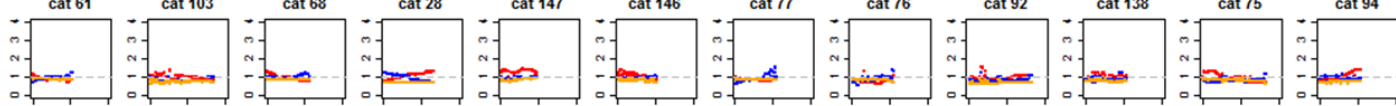

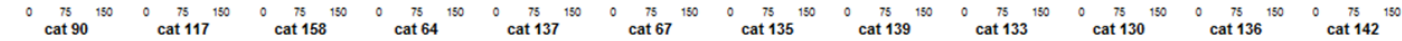

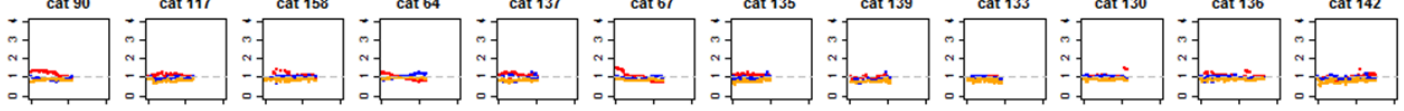

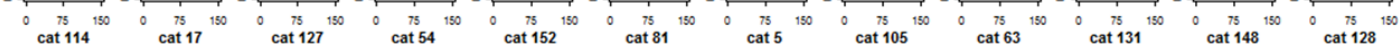

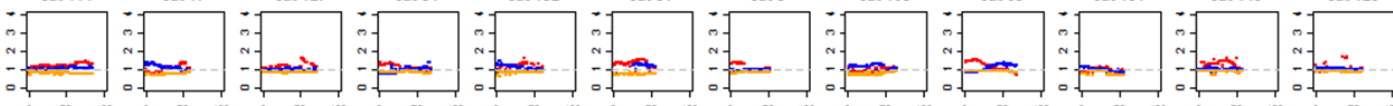

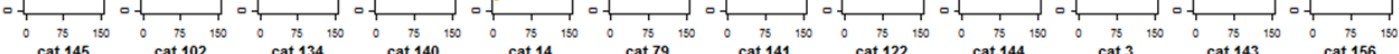

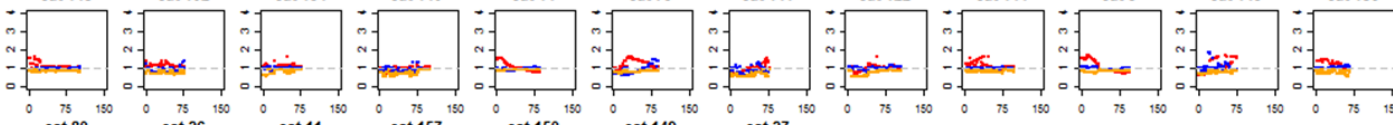

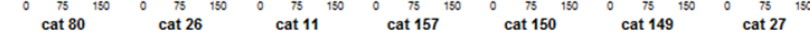

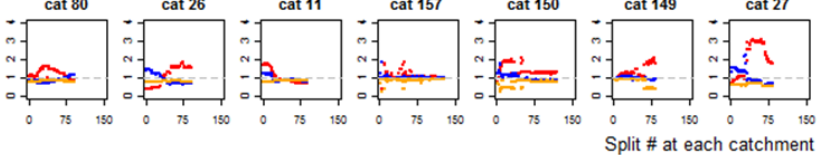

: Evaluation KGE $r$ 
[16] Confidential manuscript submitted to replace this text with name of AGU journal

Figure A2. Evaluation KGE $r, \beta$ and $\gamma$ of CMD, organized by catchment in each panel and plotted against the corresponding split number at each catchment. The horizontal grey dotted lines mark where KGE $r, \beta$ or $\gamma$ equals 1 (i.e. perfect match to observations). Catchments are sorted by increasing variance of overall evaluation KGE scores. 

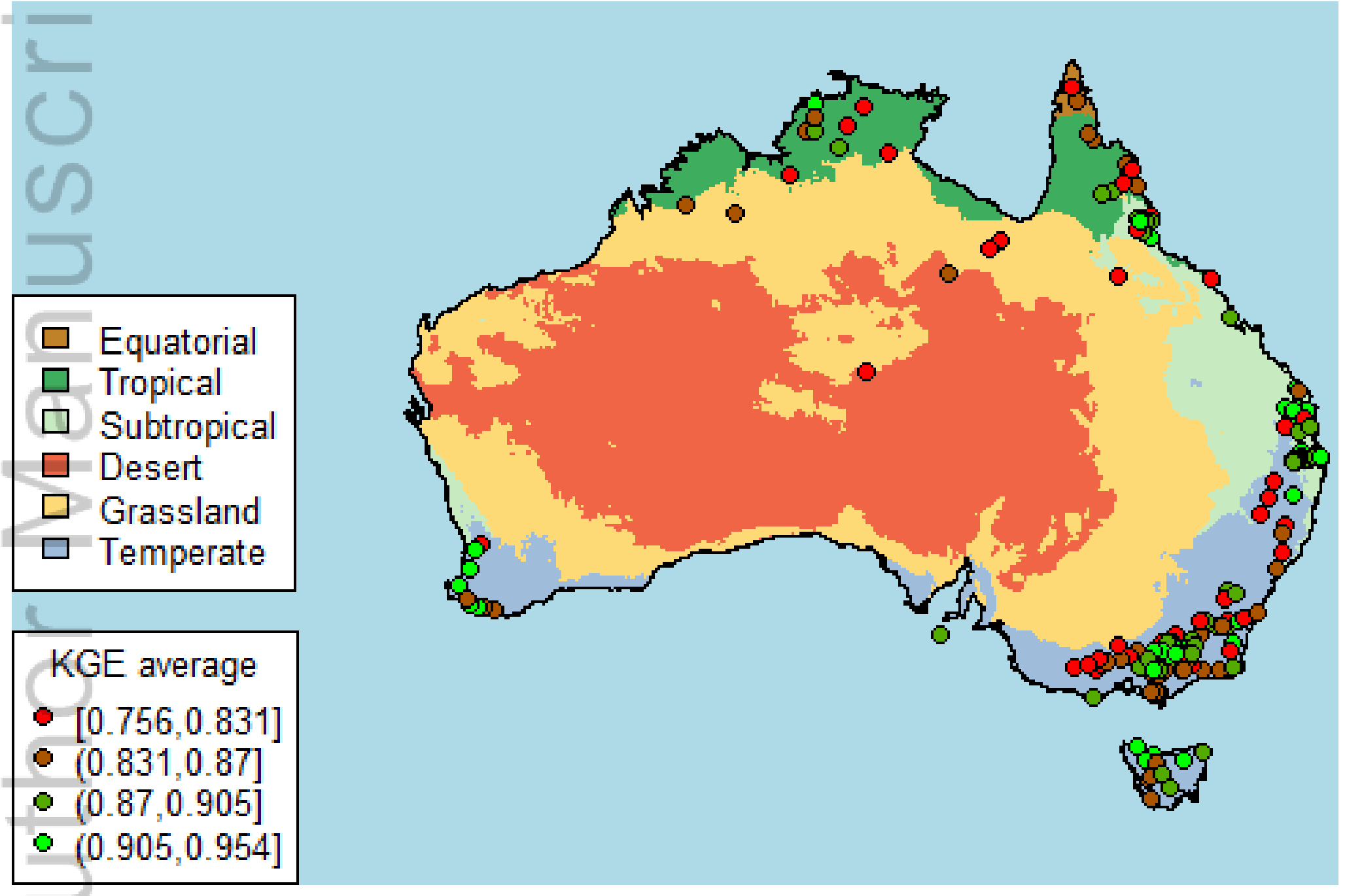

2019WR026752-f01-z-.png

This article is protected by copyright. All rights reserved. 

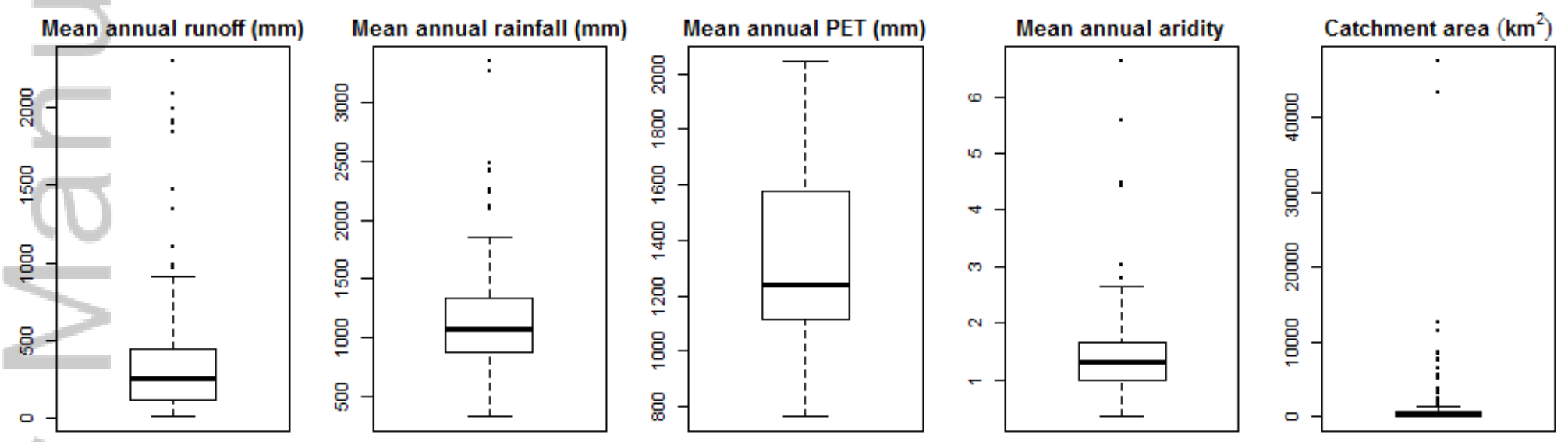

2019WR026752-f02-z-.png

This article is protected by copyright. All rights reserved. 


\section{Split-sample Calibration}

\section{Calibration split\#1, $n / 2$ years Evaluation split \#1, $n / 2$ years}

Calibration split\#2, n/2 years Evaluation split\#2, n/2years

tre

3 months

Evaluation split \#k, $n / 2$ years Calibration split \#k, $n / 2$ years

2019WR026752-f03-z-.png

This article is protected by copyright. All rights reserved. 

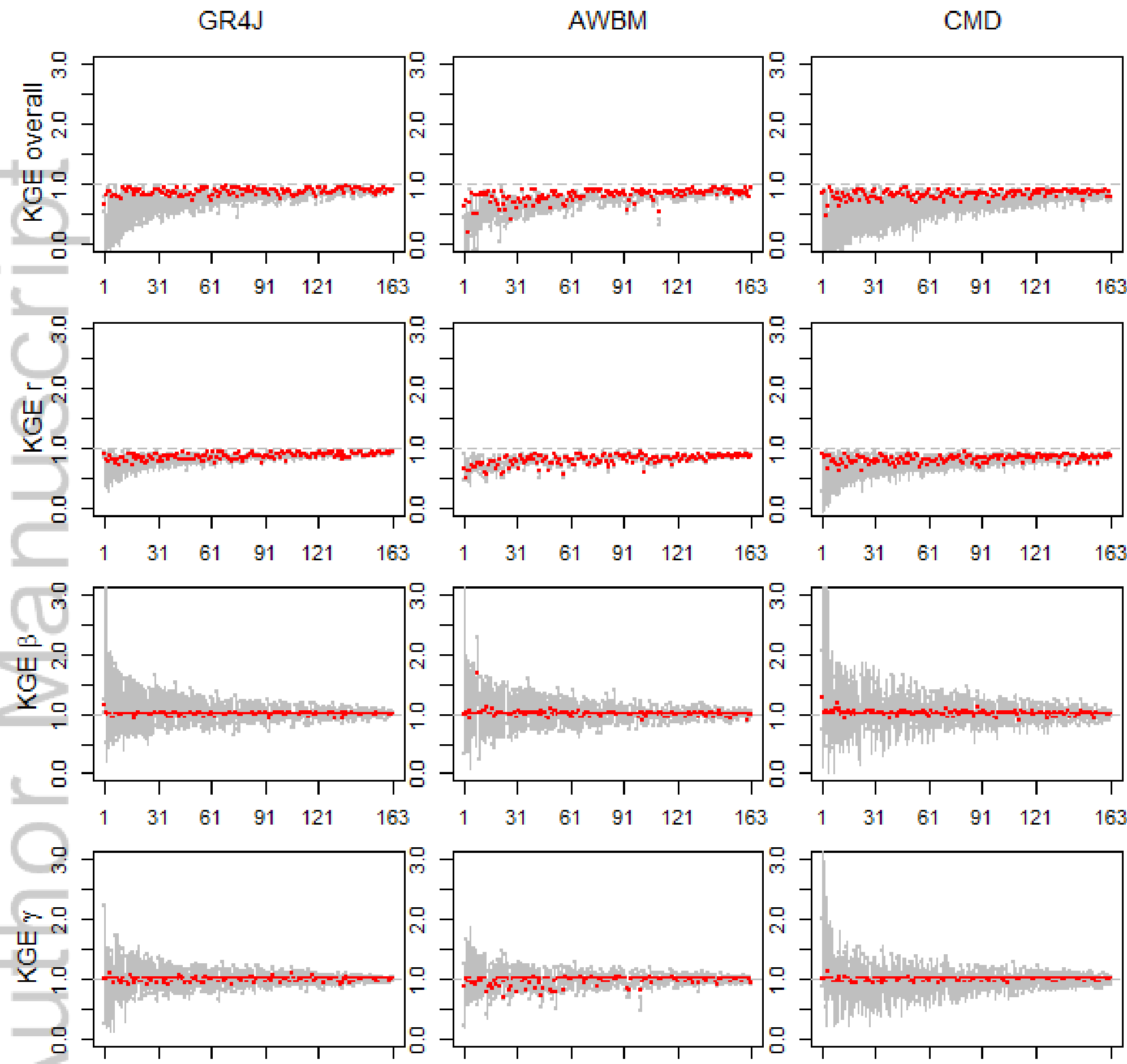

$\begin{array}{llllll}1 & 31 & 61 & 91 & 121 & 163\end{array}$

$\begin{array}{llllll}1 & 31 & 61 & 91 & 121 & 163\end{array}$

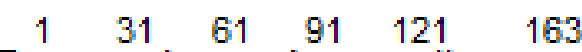
catchment index (sorted by range of correponding KGE score in each panel)

\begin{tabular}{|c|c|}
\hline+ & Average performance (calbration) - Range of performance (evaluation) \\
\hline
\end{tabular}



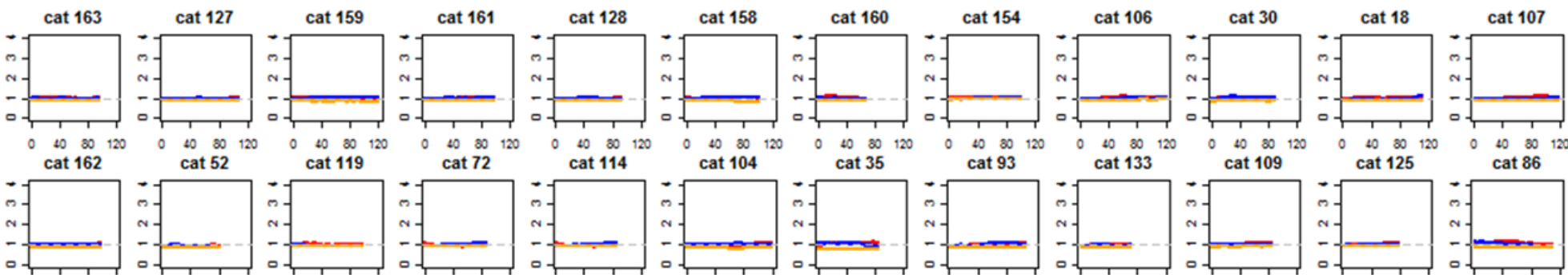

$40 \quad 30$

- $40 \quad 30120 \quad 1$
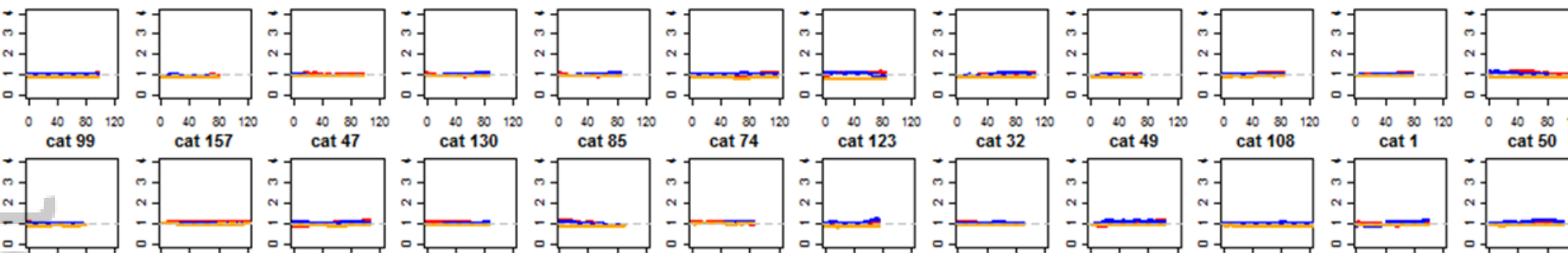

$\begin{array}{ll}40 \quad 80 \quad 120 & 1203\end{array}$
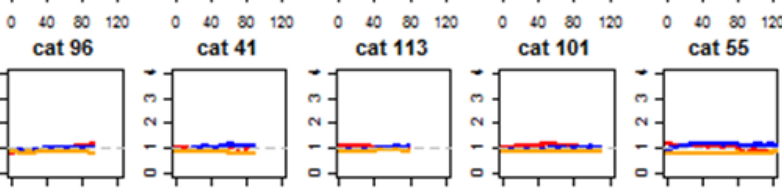

cat 39
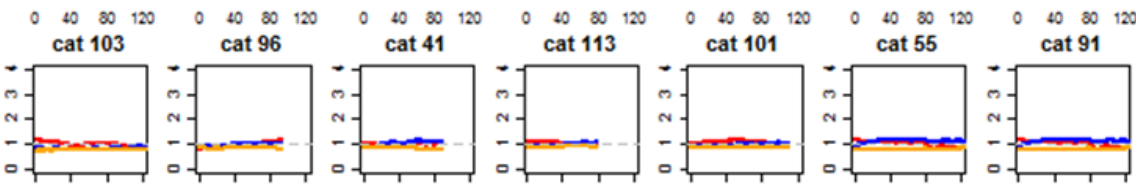

$40 \quad 80$
cat 116
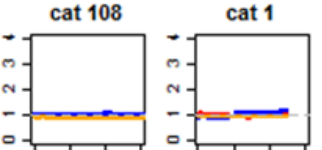

cat 50

0 ( 10,120
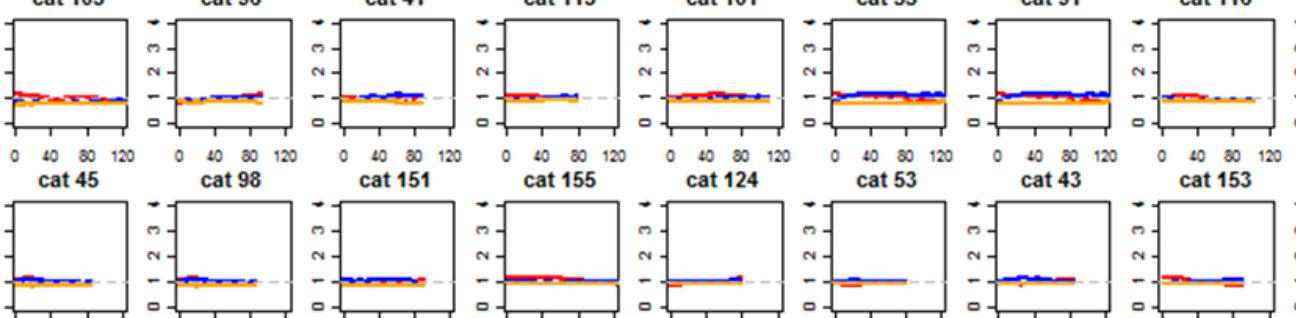

cat 66
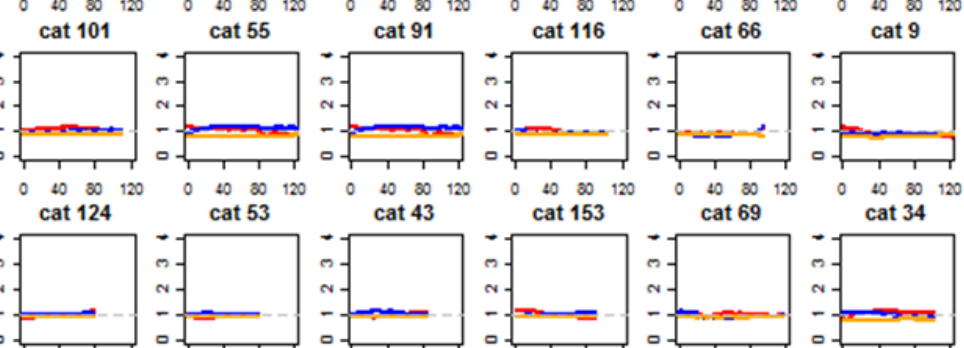

cat 34

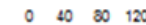

cat 40
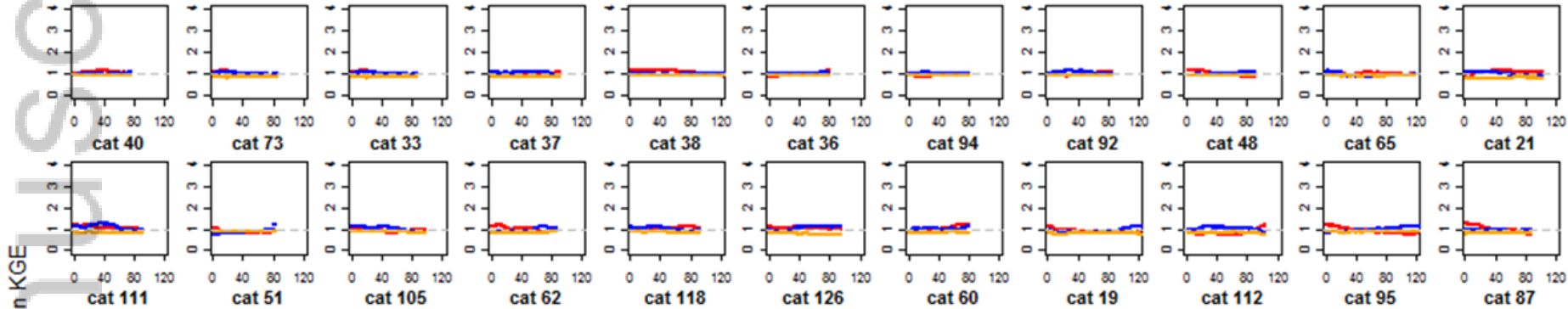

cat 105

cat 62
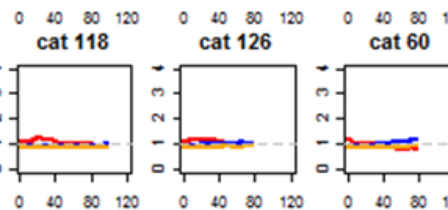

$40 \quad 80$
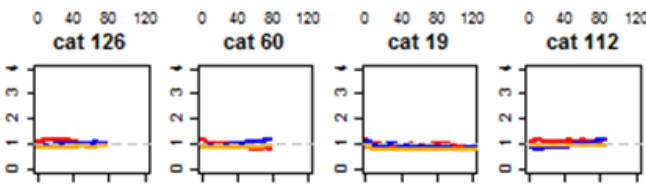

- $40 \quad 30 \quad 120$

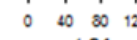

cat 21

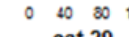

cat 22

cat 100

cat 20

cat 152

cat 115

cat 44

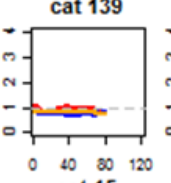

cat 71
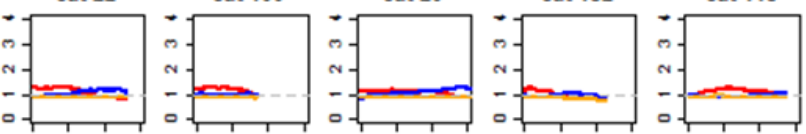

- $40 \quad 301$

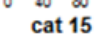

$\begin{array}{ll}40 & 80 \\ \text { cat } 81\end{array}$

$40 \quad 80$
cat 59

cat 136

$40 \quad 80$
cat 120

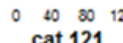

cat 23

cat 6

-
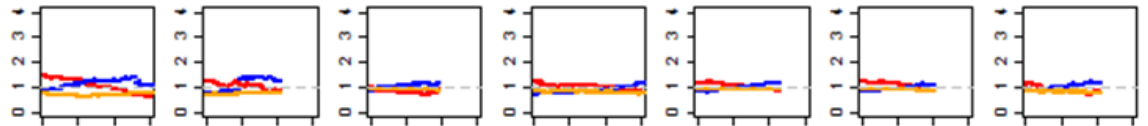

$40 \quad 20$

cat 75

cat 24

cat 88

$\begin{array}{ll}40 & 80 \\ \text { cat } 42\end{array}$

$40 \quad 80$
cat 146

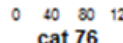

cat 5

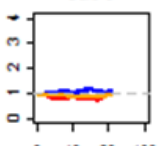

$40 \quad 80$
cat 57
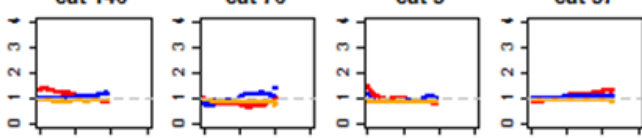

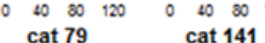

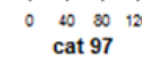

cat 150
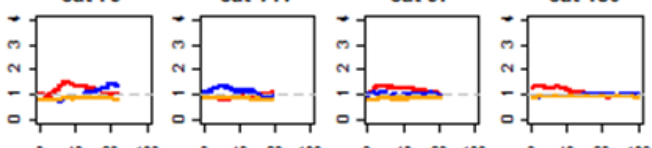

- $40 \quad 20$

$0.40 \quad 30120$
cat 77

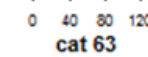

$40 \quad 80$
cat 54
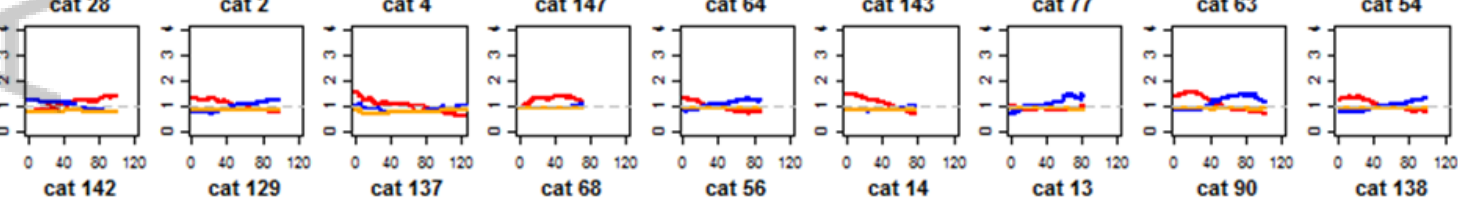

$40 \quad 80$
cat 138

$=$
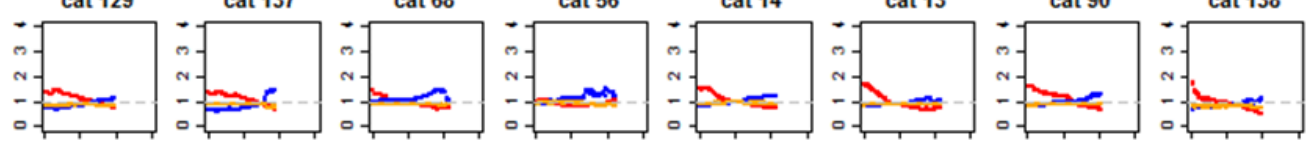

$40 \quad 80$
cat 27
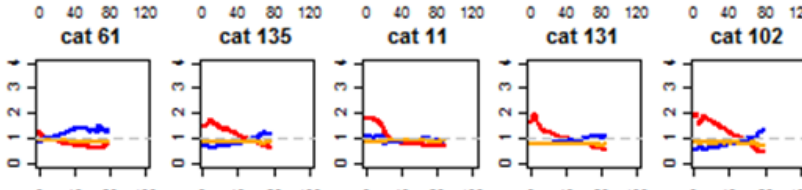

- $40 \quad 80$

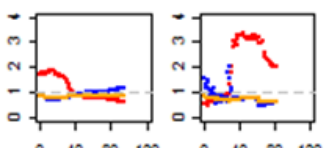

Split \# at each catchment 
Catchment 163
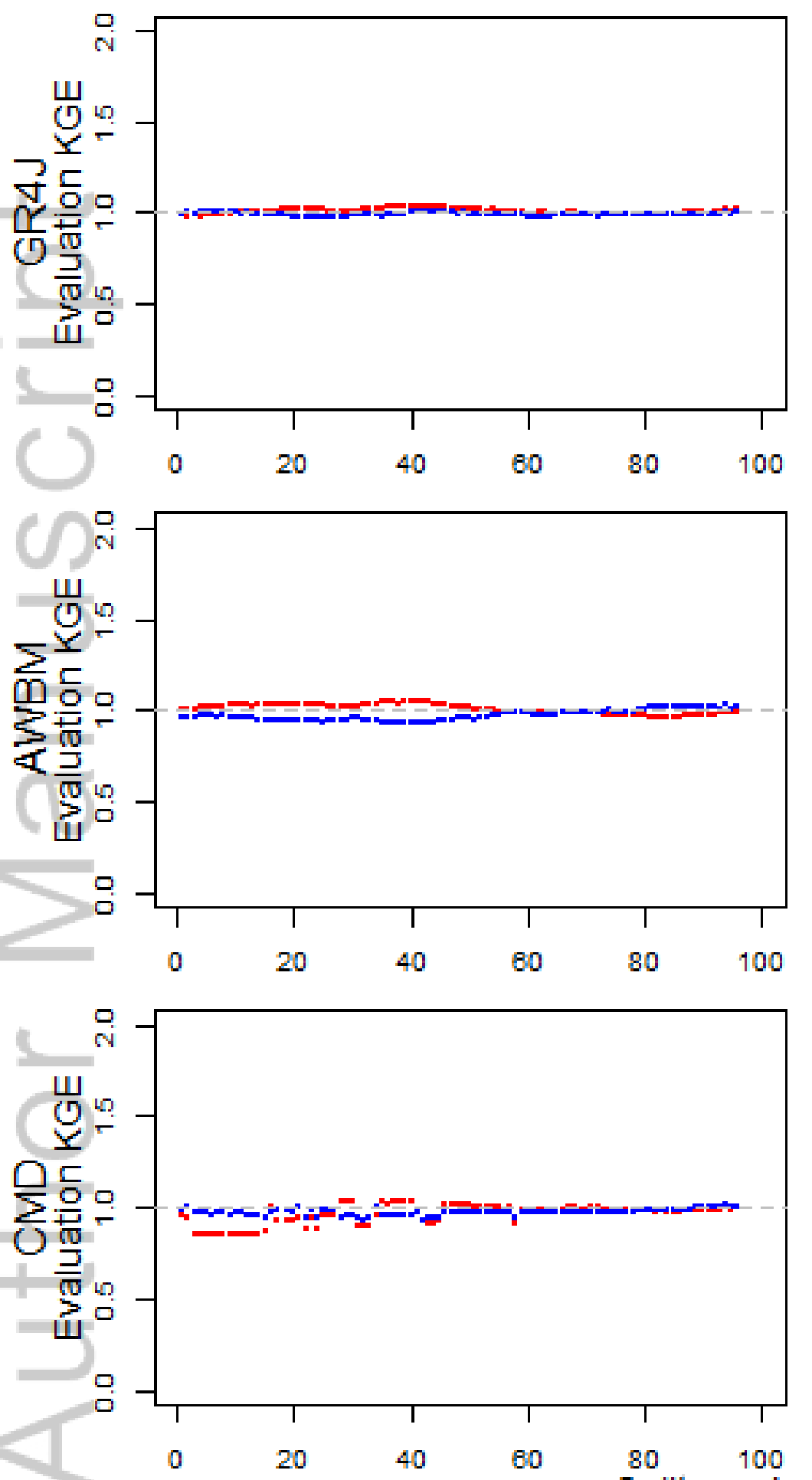
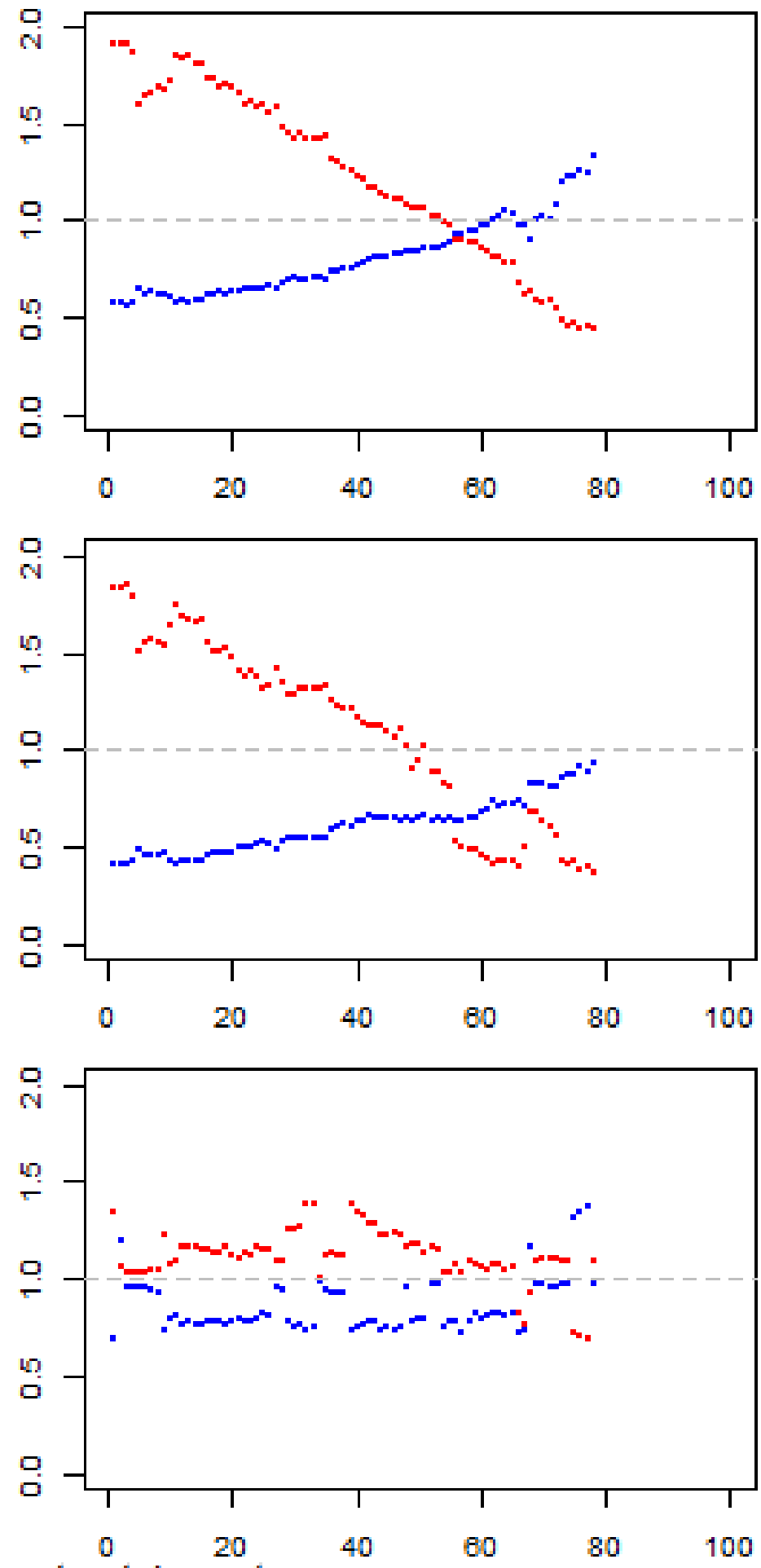

Split number at catchment

+ Evaluation KGE $\beta$ + Evaluation KGE $\gamma$ 

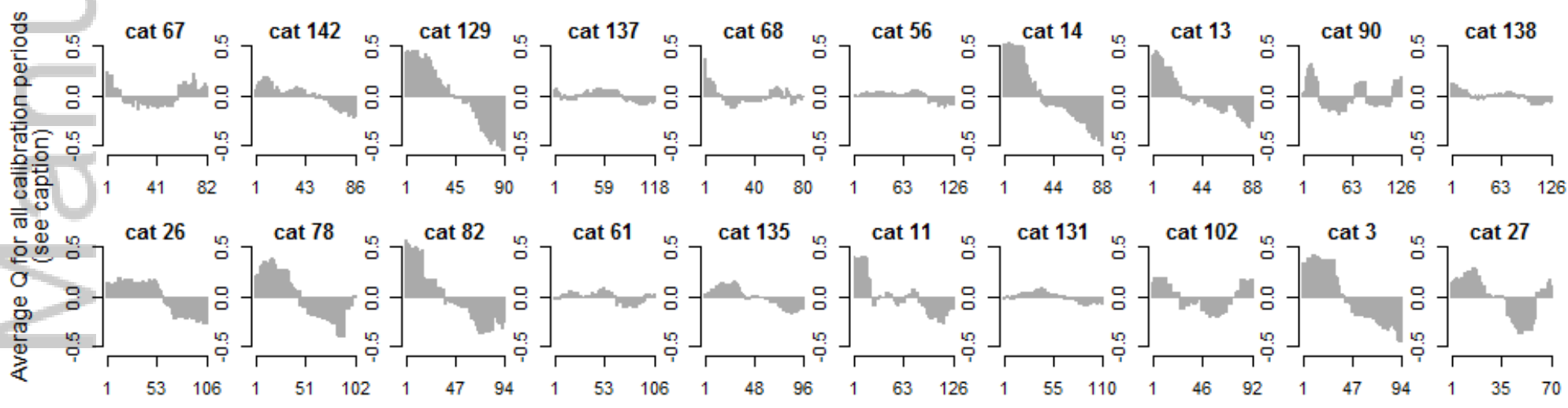

Split \# at each catchment

2019WR026752-f08-z-.png

This article is protected by copyright. All rights reserved. 


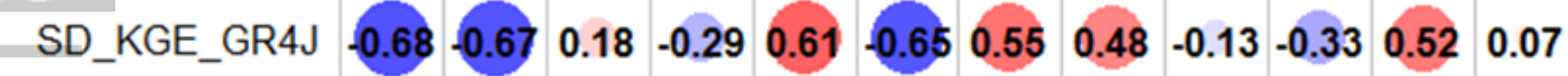
\begin{tabular}{l|l|l|l|l|l|l|l|l|l|l|l|l|l|l|l|l}
\hline SD_KGE_AWBM & -0.68 & -0.67 & 0.1 & -0.27 & 0.57 & -0.67 & 0.51 & 0.43 & -0.2 & -0.39 & 0.52 & 0.1
\end{tabular} \begin{tabular}{rl|l|l|l|l|l|l|l|l|l|l|l|}
\hline SD_KGE_CMD & -0.46 & -0.45 & -0.28 & -0.07 & 0.19 & -0.43 & 0.13 & 0.12 & -0.44 & -0.42 & 0.12 & 0.1 \\
\hline Mean_runoff & 0.91 & -0.11 & 0.37 & 0.79 & 0.98 & -0.6 & -0.65 & 0.55 & 0.75 & -0.46 & -0.37 \\
\hline
\end{tabular} $\begin{array}{llllllllllll}\text { Mean_rainfall } & -0.01 & 0.31 & 0.87 & 0.83 & -0.52 & -0.69 & 0.41 & 0.67 & -0.48 & -0.37\end{array}$

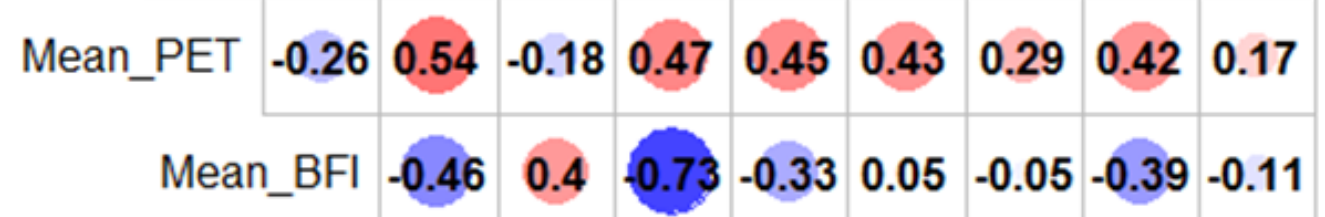
Mean_aridity $\begin{array}{cccccccc}0.77 & 0.68 & 0.84 & -0.12 & -0.36 & 0.61 & 0.44\end{array}$ Mean_RR_ratio $\begin{array}{rllllll}0.64 & -0.64 & 0.54 & 0.71 & -0.48 & -0.35\end{array}$ \begin{tabular}{l|l|l|l|l|l|} 
SD_BFI & 0.6 & 0.02 & -0.14 & 0.6 & 0.05
\end{tabular} \begin{tabular}{l|lll|l|l|l|l} 
SD_aridity & -0.02 & -0.28 & 0.61 & 0.33
\end{tabular} \begin{tabular}{ll|l|l|l|} 
SD_RR_ratio & 0.8 & 0.25 & -0.31
\end{tabular} \begin{tabular}{l|l|l|} 
SD_runoff & 0.13 & -0.33
\end{tabular} Skew_runoff 0 


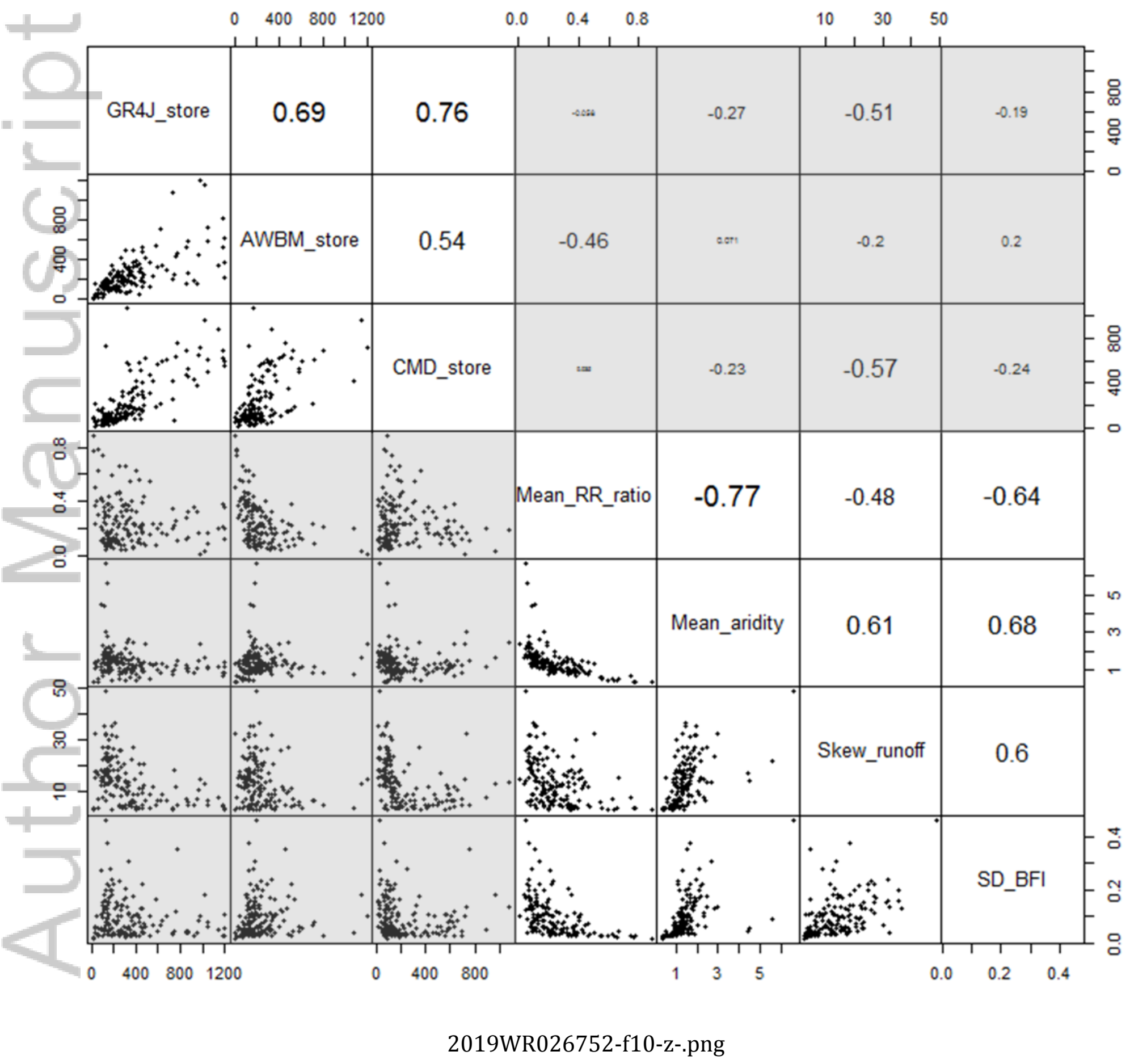

This article is protected by copyright. All rights reserved. 
KGE overall
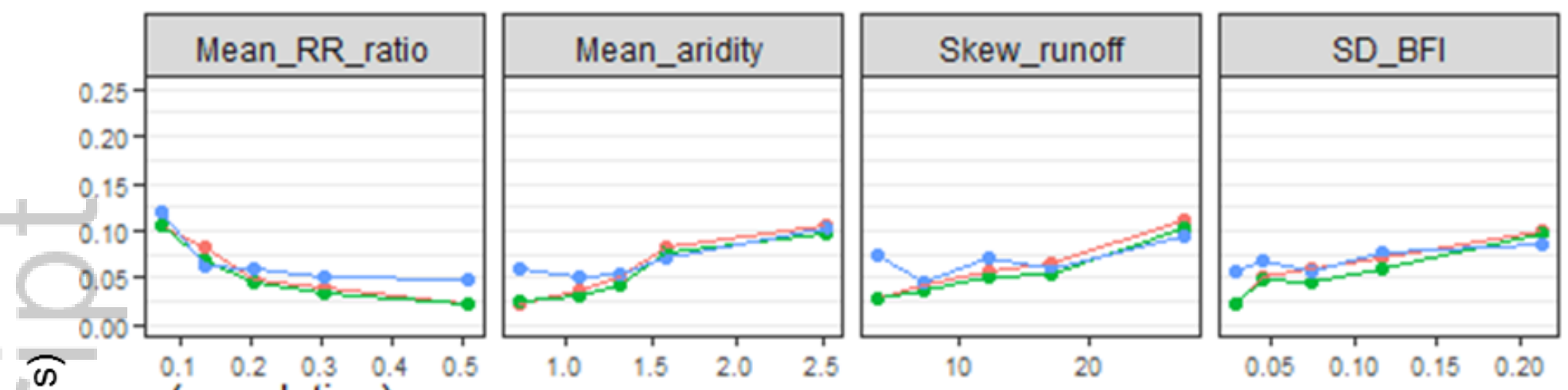

$r$ (correlation)

.
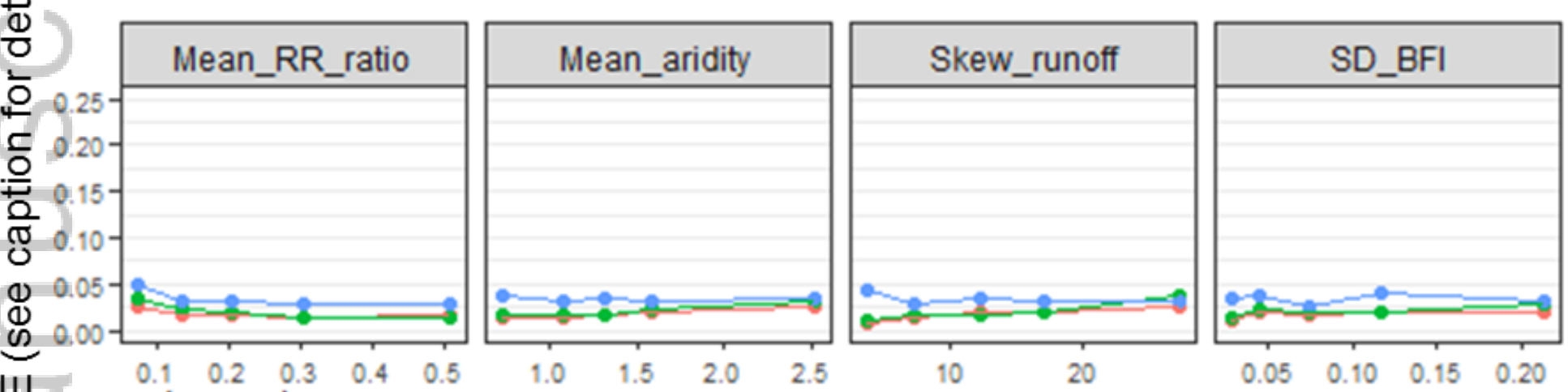

प्र $\beta$ (mean)

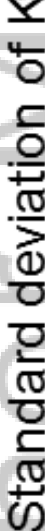
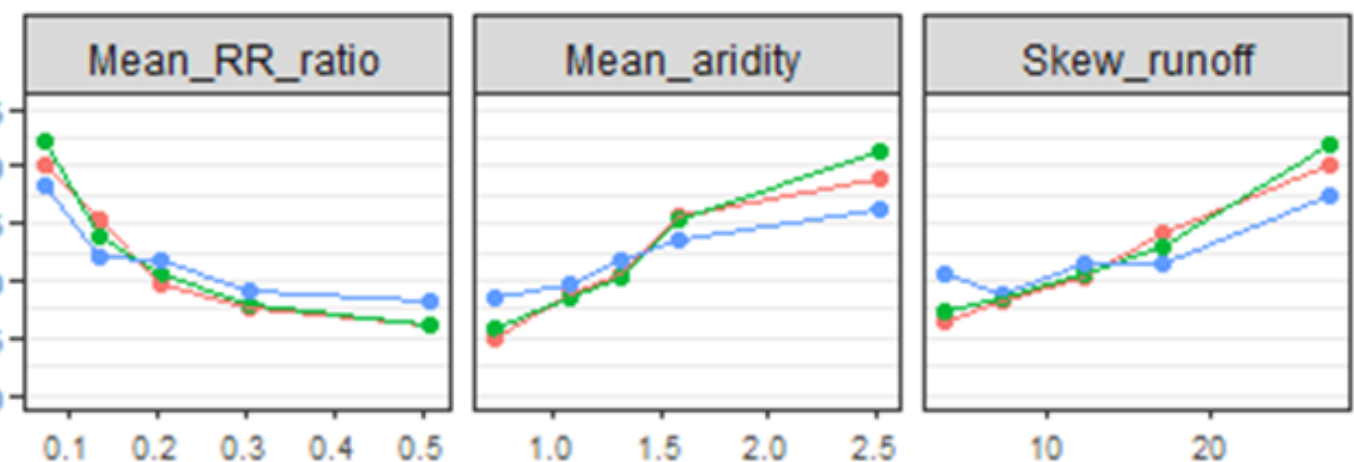

$\gamma$ (variability)
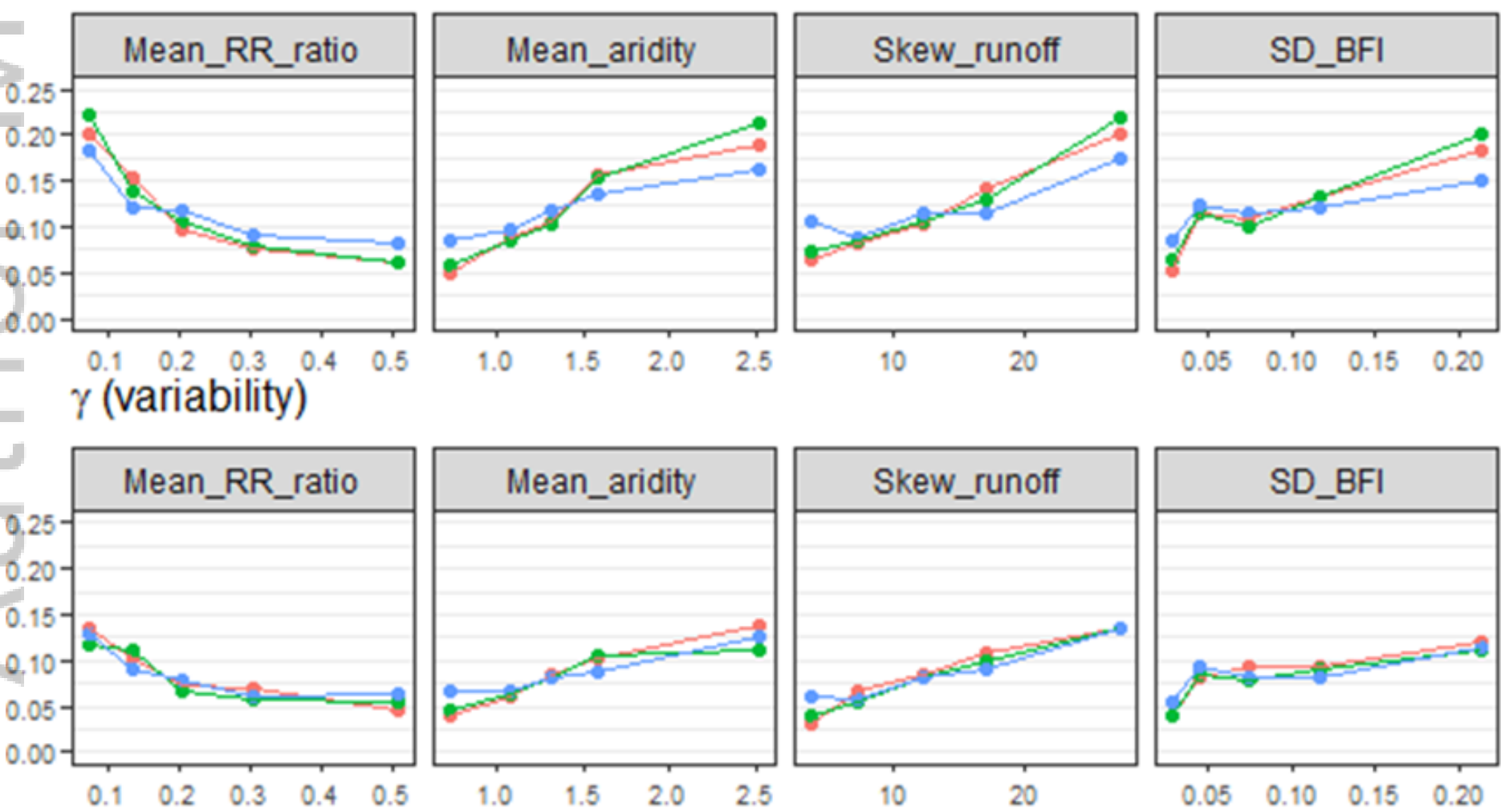

$\because$ GR4J $\rightarrow$ AWBM $\rightarrow$ CMD

2019WR026752-f11-z-.png

This article is protected by copyright. All rights reserved. 


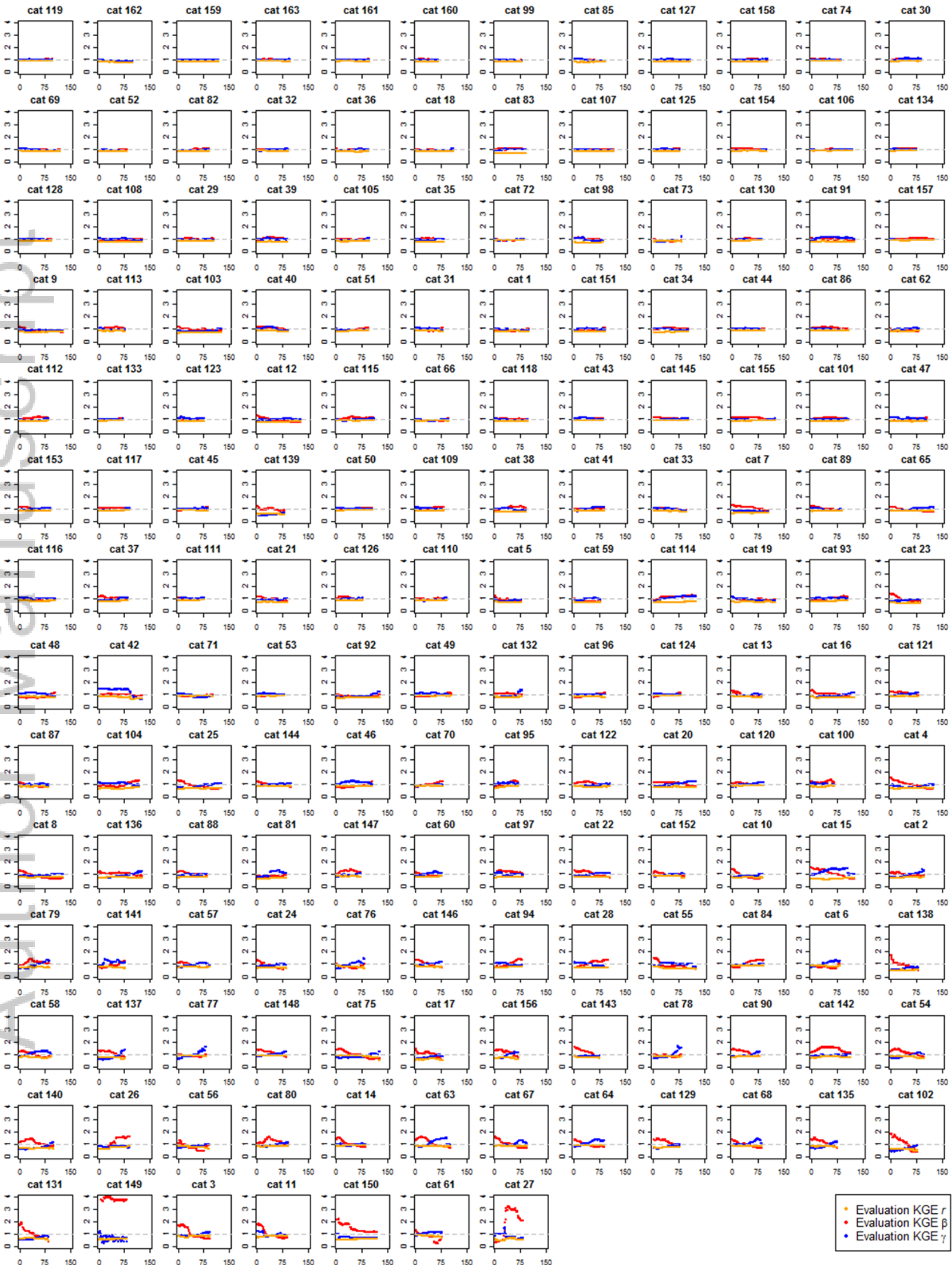



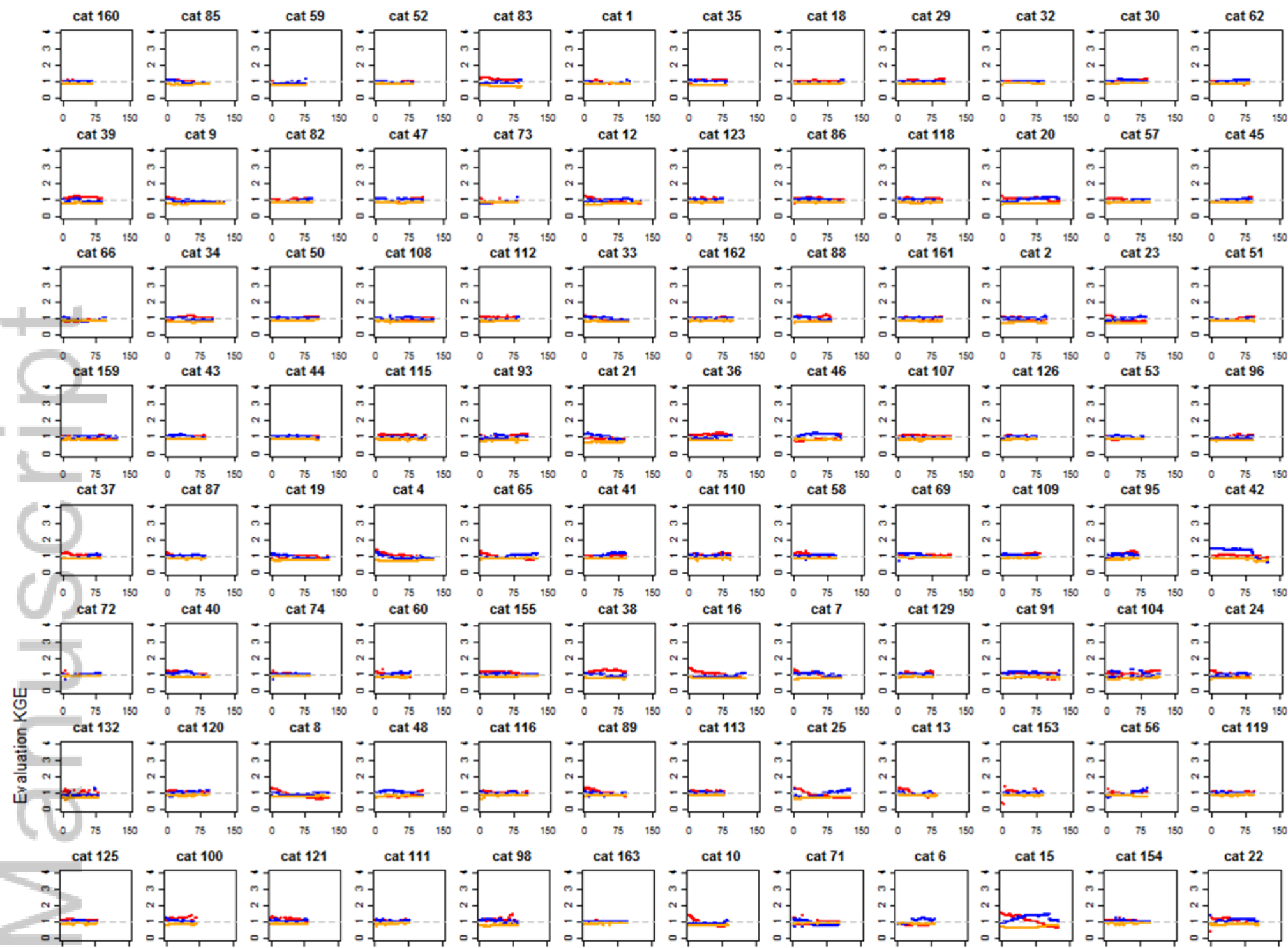

cat 24
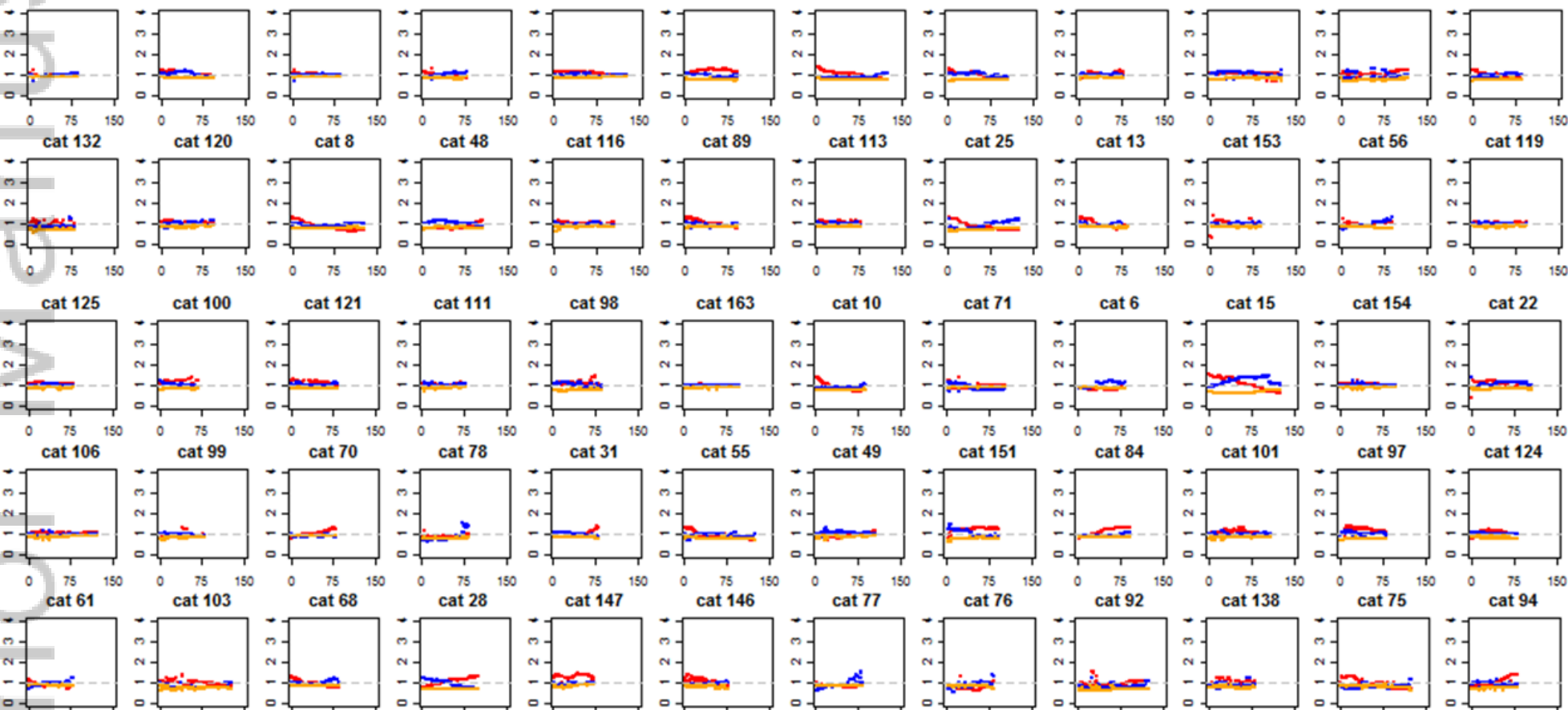

$\begin{array}{cc} & 75 \\ \text { cat } 90\end{array}$

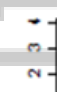

af

$-1 \rightarrow$
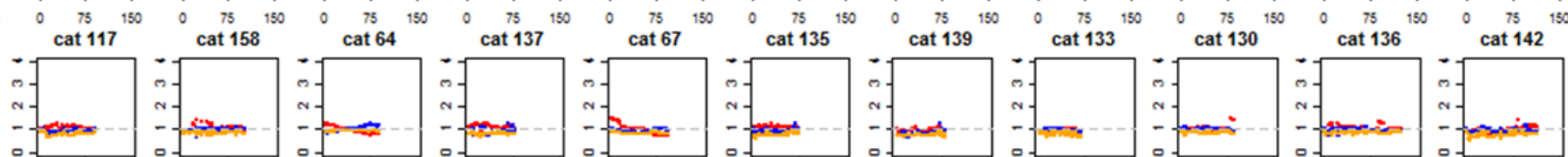

0. $75{ }^{150}$
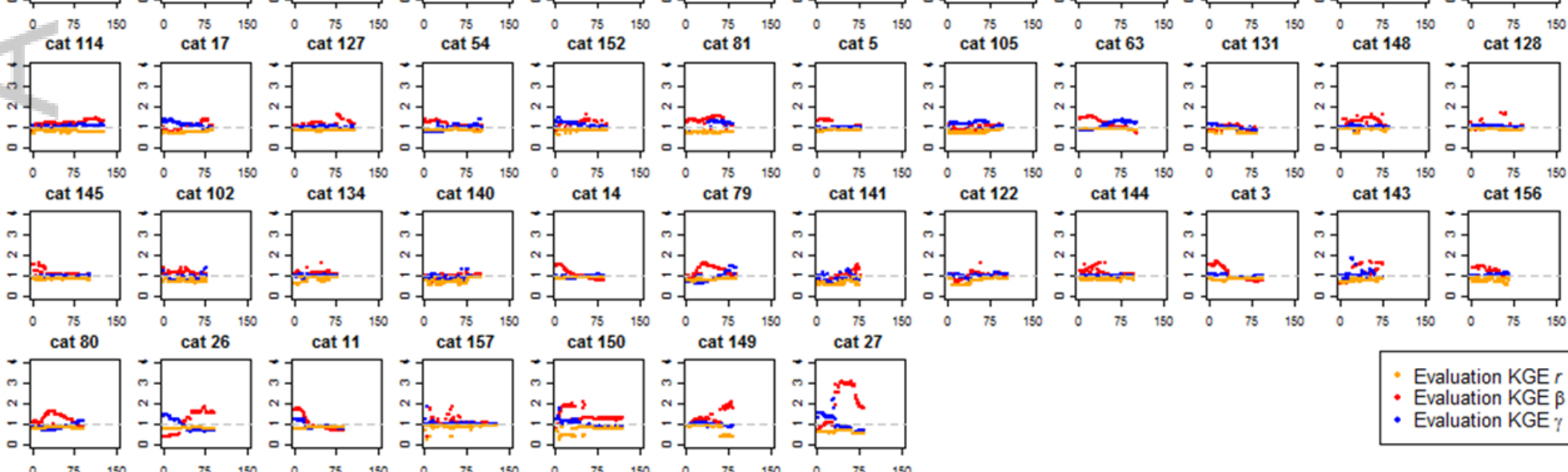

Split \# at each catchment 


\section{University Library}

\section{- M M N E R VA A gateway to Melbourne's research publications}

Minerva Access is the Institutional Repository of The University of Melbourne

Author/s:

Guo, D;Zheng, F;Gupta, H;Maier, HR

Title:

On the Robustness of Conceptual Rainfall-Runoff Models to Calibration and Evaluation Data Set Splits Selection: A Large Sample Investigation

Date:

2020-03-01

Citation:

Guo, D., Zheng, F., Gupta, H. \& Maier, H. R. (2020). On the Robustness of Conceptual Rainfall-Runoff Models to Calibration and Evaluation Data Set Splits Selection: A Large Sample Investigation. WATER RESOURCES RESEARCH, 56 (3), https:// doi.org/10.1029/2019WR026752.

Persistent Link:

http://hdl.handle.net/11343/275483 